Proyecciones Journal of Mathematics Vol. 41, $\mathrm{N}^{\circ}$ 1, pp. 45-82, February 2022.

Universidad Católica del Norte

Antofagasta - Chile

\title{
Fuzzification of strongly and locally strongly compact spaces
}

\author{
O. R. Sayed \\ Assiut University, Egypt \\ and \\ Adem Kiliçman \\ University Putra Malaysia, Malaysia \\ Received : June 2021. Accepted : July 2021
}

\begin{abstract}
In this paper, some characterizations of fuzzifying strong compactness are given, including characterizations in terms of nets and presubbases. Several characterizations of locally strong compactness in the framework of fuzzifying topology are introduced and the mapping theorems are obtained.
\end{abstract}

Keywords and Phrases: Lukasiewicz logic; semantics; fuzzifying topology; fuzzifying compactness; strong compactness; fuzzifying locally compactness; locally strong compactness.

2000 Mathematics Subject Classification: $54 A 40,54 B 10,54 D 30$. 


\section{Introduction and Preliminaries}

In the last few years fuzzy topology, as an important research field in fuzzy set theory, has been developed into a quite mature discipline [8-10, 13-14, 29]. In contrast to classical topology, fuzzy topology is endowed with richer structure, to a certain extent, which is manifested with different ways to generalize certain classical concepts. So far, according to Ref. [9], the kind of topologies defined by Chang [4] and Goguen [6] is called the topologies of fuzzy subsets, and further is naturally called $L$-topological spaces if a lattice $L$ of membership values has been chosen. Loosely speaking, a topology of fuzzy subsets (resp. an $L$-topological space) is a family $\tau$ of fuzzy subsets (resp. $L$-fuzzy subsets) of nonempty set $X$, and $\tau$ satisfies the basic conditions of classical topologies [12]. On the other hand, Höhle in [7] proposed the terminology $L$-fuzzy topology to be an $L$-valued mapping on the traditional powerset $2^{X}$ of $X$. The authors in [11, 13-14,20] defined an $L$-fuzzy topology to be an $L$-valued mapping on the $L$-powerset $L^{X}$ of $X$. In 1952, Rosser and Turquette [21] proposed emphatically the following problem: If there are many-valued theories beyond the level of predicates calculus, then what are the detail of such theories? As an attempt to give a partial answer to this problem in the case of point set topology, Ying in 1991-1993 [30-32] used a semantical method of continuous-valued logic to develop systematically fuzzifying topology. Briefly speaking, a fuzzifying topology on a set $X$ assigns each crisp subset of $X$ to a certain degree of being open, other than being definitely open or not. Roughly speaking, the semantical analysis approach transforms formal statements of interest, which are usually expressed as implication formulas in logical language, into some inequalities in the truth value set by truth valuation rules, and then these inequalities are demonstrated in an algebraic way and the semantic validity of conclusions is thus established.There are already more than 100 papers in fuzzifying topology published in the last two decades, I guess. But only a few papers can properly use the semantic method introduced in the original papers of Ying, which I strongly believe, can provide more delicate characterization of fuzzifying topological structure. So far, there has been significant research on fuzzifying topologies [1-3, 17-18, 2324]. For example, Ying [33] introduced the concepts of compactness and established a generalization of Tychonoff's theorem in the framework of fuzzifying topology. In [24] the concept of local compactness in fuzzifying topology is introduced and some of its properties are established. Generalized open sets play a very important role in General Topology and they are 
now the research topics of many topologists worldwide. Indeed a significant theme in General Topology and Real Analysis is the study of variously modified forms of continuity, separation axioms etc. by utilizing generalized open sets. One of the most well known notions and also an inspiration source is the notion of pre-open [15] sets introduced by Mashhour, Abd El-Monsef and El-Deeb in 1982. In 1984, the authors in [16] considered a strong versions of compact spaces and locally compact spaces defined in terms of pre-open subsets of a topological space. Alternative characterizations of such spaces were obtained. The concept of mixed fuzzy topological spaces is studied and investigated in some papers (see for example, [6, 21, $29,32-35]$ In [2] the concepts of fuzzifying pre-open sets and fuzzifying pre-continuity were introduced and studied. Also, the authors in [3] introduced some concepts of fuzzifying pre-separation axioms and clarified the relations of these axioms with each other as well as the relations with other fuzzifying separation axioms. Furthermore, in [1], Abd El-Baki and Sayed characterized the concepts of fuzzifying pre-irresolute functions and used the finite intersection property to give a characterization of fuzzifying strong compact spaces. In this paper, the concepts of pre-base and presubbase of fuzzifying $P$-topology are introduced. Other characterizations of fuzzifying strong compactness are given, including characterizations in terms of nets and pre-subbase. Several characterizations of locally strong compactness in the framework of fuzzifying topology are introduced and the mapping theorems are obtained. Thus we fill a gap in the existing literature on fuzzifying topology. We use the terminologies and notations in $[1-3,30-33]$ without any explanation. We note that the set of truth values is the unit interval and we do often not distinguish the connectives and their truth value functions and state strictly our results on formalization as Ying does. We will use the symbol $\otimes$ instead of the second "AND" operation $\wedge$ as dot is hardly visible. This mean that

$$
[P] \leq[\varphi \rightarrow \psi] \Leftrightarrow \quad[P] \otimes[\varphi] \leq[\psi] .
$$

All of the contributions in General Topology in this paper which are not referenced may be original.

We procure some useful definitions and results which are useful in the rest of the present paper.

A unary fuzzy predicate $\tau_{P}: 2^{X} \rightarrow[0,1]$, called fuzzifying pre-open [2], is given as follows:

$$
\left.A \in \tau_{P}:=\forall x(x \in A \rightarrow x \in \operatorname{Int}(C l(A))) \text {, i.e., } \tau_{P}(A)=\bigwedge_{x \in A} \operatorname{Int}(C l(A))(x)\right) .
$$


Similarly, A unary fuzzy predicate $\mathcal{F}_{P}: 2^{X} \rightarrow[0,1]$, called fuzzifying pre-closed [2], is given as follows: $A \in \mathcal{F}_{P}:=X-A \in \tau_{P}$. The fuzzifying pre-neighborhood system of a point $x \in X[2]$ is denoted by $N_{x}^{P^{X}}$ (or $\left.N_{x}^{P}\right): 2^{X} \rightarrow[0,1]$ and defined as $N_{x}^{P}(A)=\bigvee_{x \in B \subseteq A} \tau_{P}(B)$. The fuzzifying pre-closure of a set $A \subseteq X[2]$, denoted by $C l_{P} \in[0,1]^{X}$, is defined as $C l_{P}(A)(x)=1-N_{x}^{P}(X-A)$. If $(X, \tau)$ is a fuzzifying topological space and $N(X)$ is the class of all nets in $X$, then the binary fuzzy predicates $\triangleright^{P}, \propto^{P}: N(X) \times X \rightarrow[0,1][23]$ are defined as

$$
S \triangleright^{P} x:=\forall A\left(A \in N_{x}^{P^{X}} \rightarrow S \widetilde{\subset} A\right), \quad S \propto^{P} x:=\forall A\left(A \in N_{x}^{P^{X}} \rightarrow S \widetilde{\complement} A\right),
$$

where " $S \triangleright^{P} x ", " S \propto^{P} x "$ stand for $" S$ pre-converges to $x ", " x$ is a preaccumulation point of $S$ ", respectively; and " $\widetilde{\complement}$, " $\widetilde{\complement} "$ are the binary crisp predicates "almost in "," often in", respectively. The degree to which $x$ is a pre-adherence point of $S$ is $a d h_{P} S(x)=\left[S \propto^{P} x\right]$. If $(X, \tau)$ and $(Y, \sigma)$ are two fuzzifying topological spaces and $f \in Y^{X}$, the unary fuzzy predicates $C_{P}, I_{P}: Y^{X} \rightarrow[0,1]$, called fuzzifying pre-continuity [2], fuzzifying preirresoluteness [1], are given as

$C_{P}(f):=\forall B\left(B \in \sigma \rightarrow f^{-1}(B) \in \tau_{P}\right), I_{P}(f):=\forall B\left(B \in \sigma_{P} \rightarrow f^{-1}(B) \in \tau_{P}\right)$,

respectively. Let $\Omega$ be the class of all fuzzifying topological spaces. A unary fuzzy predicate $T_{2}^{P}: \Omega \rightarrow[0,1]$, called fuzzifying pre-Hausdorffness [3], is given as follows:

$T_{2}^{P}(X, \tau)=\forall x \forall y\left((x \in X \wedge y \in X \wedge x \neq y) \rightarrow \exists B \exists C\left(B \in N_{x}^{P} \wedge C \in N_{y}^{P} \wedge B \cap C \equiv \phi\right)\right)$.

A unary fuzzy predicate $\Gamma: \Omega \rightarrow[0,1]$, called fuzzifying compactness [33], is given as follows:

$$
\Gamma(X, \tau):=(\forall \Re)\left(K_{\circ}(\Re, X) \longrightarrow(\exists \wp)((\wp \leq \Re) \wedge K(\wp, A) \otimes F F(\wp))\right)
$$

and if $A \subseteq X$, then $\Gamma(A):=\Gamma(A, \tau / A)$. For $K, K_{\circ}$ (resp. $\leq$ and $\left.F F\right)$ see [31, Definition 4.4] (resp. [31, Theorem 4.3] and [33, Definition 1.1 and Lemma 1.1]). A unary fuzzy predicate $f I:[0,1]^{2^{X}} \rightarrow[0,1]$, called fuzzy finite intersection property [33], is given as

$$
f I(\Re):=\forall \wp((\wp \leq \Re) \wedge F F(\wp) \rightarrow \exists x \forall B(B \in \wp \rightarrow x \in B)) .
$$

A fuzzifying topological space $(X, \tau)$ is said to be fuzzifying $P$-topological space [1] if $\tau_{P}(A \cap B) \geq \tau_{P}(A) \wedge \tau_{P}(B)$. A binary fuzzy predicate 
$K_{P}:[0,1]^{2^{X} \times 2^{X}} \rightarrow[0,1]$, called fuzzifying pre-open covering [1], is given as $K_{P}(\Re, A):=K(\Re, A) \otimes\left(\Re \subseteq \tau_{P}\right)$. A unary fuzzy predicate $\Gamma_{P}: \Omega \rightarrow[0,1]$, called fuzzifying strongly compactness [1], is given as follows:

$$
(X, \tau) \in \Gamma_{P}:=(\forall \Re)\left(K_{P}(\Re, X) \longrightarrow(\exists \wp)((\wp \leq \Re) \wedge K(\wp, X) \otimes F F(\wp))\right)
$$

and if $A \subseteq X$, then $\Gamma_{P}(A):=\Gamma_{P}(A, \tau / A)$. It is obvious that

$$
\Gamma_{P}(X, \tau):=\Gamma\left(X, \tau_{P}\right), \Gamma_{P}(A, \tau / A):=\Gamma\left(A, \tau_{P} / A\right)
$$

and

$$
=K_{\circ}(\Re, A) \longrightarrow K_{P}(\Re, A) .
$$

A fuzzifying strongly compact space is a generalization of strongly compact space [16]. A space $X$ is said to be strongly compact if every pre-open cover $X$ has a finite subcover.

A unary fuzzy predicate $L C: \Omega \rightarrow[0,1]$, called fuzzifying locally compactness [24], is given as follows:

$$
(X, \tau) \in L C:=(\forall x)(\exists B)((x \in \operatorname{Int}(B) \otimes \Gamma(B, \tau / B)) .
$$

A space $X$ is said to be locally strongly compact [16] if each point of $X$ has a neighbourhood which is a strongly compact subspace.

\section{Fuzzifying pre-base and pre-subbase}

Definition 2.1. Let $(X, \tau)$ be a fuzzifying topological space and $\beta_{P} \subseteq \tau_{P}$. Then $\beta_{P}$ is called a pre-base of $\tau_{P}$ if $\beta_{P}$ fulfils the condition:

$$
\models A \in N_{x}^{P^{X}} \rightarrow \exists B\left(\left(B \in \beta_{P}\right) \wedge(x \in B \subseteq A)\right) .
$$

Example 2.1. Let $X=\{a, b, c\}$, and $I=[0,1]$. Define a mapping $\tau$ : $P(X) \longrightarrow I$ on $X$ as follows: $\tau(\emptyset)=\tau(X)=1, \tau(\{a, c\})=0, \tau(\{a, b\})=\frac{1}{5}$, $\tau(\{b, c\})=\frac{1}{2}, \tau(\{a\})=0, \tau(\{b\})=\frac{3}{4}, \tau(\{c\})=\frac{1}{2}$. Then we can easily verify that $\tau$ is a fuzzifying topology. By calculating,

$\tau_{P}(\emptyset)=\tau_{P}(X)=1, \tau_{P}(\{a, c\})=\frac{1}{4}, \tau_{P}(\{a, b\})=1, \tau_{P}(\{b, c\})=1$, $\tau_{P}(\{a\})=\frac{1}{4}, \tau_{P}(\{b\})=\frac{3}{4}, \tau_{P}(\{c\})=1$. If we set $\beta_{P}=\tau_{P}$, then $N_{x}^{P}(A)=\bigvee_{x \in B \subseteq A} \tau_{P}(B)=\bigvee_{x \in B \subseteq A} \beta_{P}(B)$. Obviously, $\beta_{P}$ is a pre-base of $\tau_{P}$ by Definition 2.1.

Theorem 2.1. $\beta_{P}$ is a pre-base of $\tau_{P}$ if and only if $\tau_{P}=\beta_{P}^{(\cup)}$, where

$$
\beta_{P}^{(\cup)}(A)=\bigvee_{\lambda \in \Lambda} \bigwedge_{B_{\lambda}=A} \beta_{P}\left(B_{\lambda}\right) .
$$


Proof. Suppose that $\beta_{P}$ is a pre-base of $\tau_{P}$. If

$$
\bigcup_{\lambda \in \Lambda} B_{\lambda}=A
$$

then from Theorem 3.1 (1) (b) in [2],

$$
\tau_{P}(A)=\tau_{P}\left(\bigcup_{\lambda \in \Lambda} B_{\lambda}\right) \geq \bigwedge_{\lambda \in \Lambda} \tau_{P}\left(B_{\lambda}\right) \geq \bigwedge_{\lambda \in \Lambda} \beta_{P}\left(B_{\lambda}\right) .
$$

Consequently,

$$
\tau_{P}(A) \geq \bigcup_{\lambda \in \Lambda} \bigvee_{\lambda} B_{\lambda}=A \bigwedge_{\lambda \in \Lambda} \beta_{P}\left(B_{\lambda}\right)
$$

To prove that

$$
\tau_{P}(A) \geq \bigcup_{\lambda \in \Lambda} \bigvee_{\lambda} B_{\lambda}=A \bigwedge_{\lambda \in \Lambda} \beta_{P}\left(B_{\lambda}\right)
$$

we first prove

$$
\tau_{P}(A)=\bigwedge_{x \in A} \bigvee_{x \in B \subseteq A} \tau_{P}(B) .
$$

(Indeed, assume $\delta_{x}=\{B: x \in B \subseteq A\}$. Then, for any

$$
f \in \prod_{x \in A} \delta_{x}, \bigcup_{x \in A} f(x)=A,
$$

and further we have

$$
\begin{aligned}
\tau_{P}(A) & =\tau_{P}\left(\bigcup_{x \in A} f(x)\right) \\
& \geq \bigwedge_{x \in A} \tau_{P}(f(x)) \\
& \geq \bigvee_{f \in \prod_{x \in A} \bigwedge_{x}} \tau_{x \in A}(f(x)) \\
& =\bigwedge_{x \in A} \bigvee_{x \in B \subseteq A} \tau_{P}(B)
\end{aligned}
$$

Also,

$$
\tau_{P}(A) \leq \bigwedge_{x \in A} \bigvee_{x \in B \subseteq A} \tau_{P}(B)
$$


Therefore,

$$
\left.\tau_{P}(A)=\bigwedge_{x \in A} \bigvee_{x \in B \subseteq A} \tau_{P}(B)\right)
$$

Now, since

$$
\begin{aligned}
N_{x}^{P^{X}}(A) & \leq \bigvee_{x \in B \subseteq A} \beta_{P}(B), \\
\tau_{P}(A) & =\bigwedge_{x \in A} \bigvee_{x \in B \subseteq A} \tau_{P}(B)=\bigwedge_{x \in A} N_{x}^{P^{X}}(A) \\
& \leq \bigwedge_{x \in A} \bigvee_{x \in B \subseteq A} \beta_{P}(B)=\bigvee_{f \in \prod_{x \in A} \bigwedge_{\delta_{x}} \beta_{x \in A}(f(x)) .}
\end{aligned}
$$

Then,

$$
\tau_{P}(A) \leq \bigcup_{\lambda \in \Lambda} \bigwedge_{B_{\lambda}=A} \beta_{\lambda \in \Lambda}\left(B_{\lambda}\right)
$$

Therefore,

$$
\tau_{P}(A)=\bigvee_{\lambda \in \Lambda} \bigwedge_{B_{\lambda}=A} \beta_{P}\left(B_{\lambda}\right)
$$

In the other side, we assume that

$$
\tau_{P}(A)=\bigvee_{\lambda \in \Lambda} \bigwedge_{B_{\lambda}=A} \beta_{P}\left(B_{\lambda}\right)
$$

and we will show that $\beta_{P}$ is a pre-base of $\tau_{P}$, i.e., for any $A \subseteq X, N_{x}^{P^{X}}(A) \leq$ $\bigvee_{x \in B \subseteq A} \beta_{P}(B)$. Indeed, if $x \in B \subseteq A$ and $\bigcup_{\lambda \in \Lambda} B_{\lambda}=B$, then there exists $\lambda_{\circ} \in \Lambda$ such that $x \in B_{\lambda_{\circ}}$ and

$$
\bigwedge_{\lambda \in \Lambda} \beta_{P}\left(B_{\lambda}\right) \leq \beta_{P}\left(B_{\lambda_{0}}\right) \leq \bigvee_{x \in B \subseteq A} \beta_{P}(B)
$$

Therefore,

$$
\begin{aligned}
& N_{x}^{P^{X}}(A)=\bigvee_{x \in B \subseteq A} \tau_{P}(B) \\
= & \bigvee_{x \in B \subseteq A} \bigvee_{\lambda \in \Lambda} \bigwedge_{B_{\lambda}=A} \beta_{P}\left(B_{\lambda}\right)
\end{aligned}
$$




$$
\leq \bigvee_{x \in B \subseteq A} \beta_{P}(B)
$$

Theorem 2.2. Let $\beta_{P}: 2^{X} \rightarrow[0,1]$. Then $\beta_{P}$ is a pre-base for some fuzzifying $P$-topology $\tau_{P}$ if and only if it has the following properties:

(1) $\beta_{P}^{(\cup)}(X)=1$;

$(2) \models\left(A \in \beta_{P}\right) \wedge\left(B \in \beta_{P}\right) \wedge(x \in A \cap B) \rightarrow \exists C\left(\left(C \in \beta_{P}\right) \wedge(x \in C \subseteq\right.$ $A \cap B))$.

Proof. If $\beta_{P}$ is a pre-base for some fuzzifying $P$-topology $\tau_{P}$, then $\tau_{P}(X)=\beta_{P}^{(\cup)}(X)$. Clearly, $\beta_{P}^{(\cup)}(X)=1$. In addition, if $x \in A \cap B$, then

$$
\begin{aligned}
\beta_{P}(A) \wedge \beta_{P}(B) \leq \tau_{P}(A) \wedge \tau_{P}(B) & \leq \tau_{P}(A \cap B) \leq N_{x}^{P^{X}}(A \cap B) \\
& \leq \bigvee_{x \in C \subseteq A \cap B} \beta_{P}(C) .
\end{aligned}
$$

Conversely, if $\beta_{P}$ satisfies (1) and (2), then we have $\tau_{P}$ is a fuzzifying $P$-topology. In fact, $\tau_{P}(X)=1$. For any $\left\{A_{\lambda}: \lambda \in \Lambda\right\} \subseteq P(X)$, we set

$$
\delta_{\lambda}=\left\{\left\{B_{\Phi_{\lambda}}: \Phi_{\lambda} \in \Lambda_{\lambda}\right\}: \bigcup_{\Phi_{\lambda} \in \Lambda_{\lambda}} B_{\Phi_{\lambda}}=A_{\lambda}\right\} .
$$

Then, for any

$$
f \in \prod_{\lambda \in \Lambda} \delta_{\lambda}, \bigcup_{\lambda \in \Lambda} \bigcup_{B_{\Phi_{\lambda}} \in f(\lambda)} B_{\Phi_{\lambda}}=\bigcup_{\lambda \in \Lambda} A_{\lambda}
$$

Therefore,

$$
\begin{aligned}
& \tau_{P}\left(\bigcup_{\lambda \in \Lambda} A_{\lambda}\right)=\bigcup_{\Phi \in \Lambda} \bigvee_{B_{\Phi}=\bigcup_{\lambda \in \Lambda}} \bigwedge_{\Phi \in \Lambda} \beta_{P}\left(B_{\Phi}\right)
\end{aligned}
$$

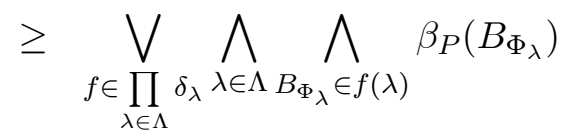

$$
\begin{aligned}
& \geq \bigwedge_{\lambda \in \Lambda} \bigvee_{\left\{B_{\Phi_{\lambda}}: \Phi_{\lambda} \in \Lambda_{\lambda}\right\} \in \delta_{\lambda}} \bigwedge_{\Phi_{\lambda} \in \Lambda_{\lambda}} \beta_{P}\left(B_{\Phi_{\lambda}}\right) \\
& =\bigwedge_{\lambda \in \Lambda} \tau_{P}\left(A_{\lambda}\right) \text {. }
\end{aligned}
$$


Finally, we need to prove that

$$
\tau_{P}(A \cap B) \geq \tau_{P}(A) \wedge \tau_{P}(B) .
$$

If $\tau_{P}(A)>t, \tau_{P}(B)>t$, then there exists

$$
\left\{B_{\lambda_{1}}: \lambda_{1} \in \Lambda_{1}\right\}, \quad\left\{B_{\lambda_{2}}: \lambda_{2} \in \Lambda_{2}\right\}
$$

such that $\bigcup_{\lambda_{1} \in \Lambda_{1}} B_{\lambda_{1}}=A, \bigcup_{\lambda_{2} \in \Lambda_{2}} B_{\lambda_{2}}=B$

and for any $\lambda_{1} \in \Lambda_{1}, \quad \beta_{P}\left(B_{\lambda_{1}}\right)>t$,

for any $\lambda_{2} \in \Lambda_{2}, \quad \beta_{P}\left(B_{\lambda_{2}}\right)>t$. Now, for any $x \in A \cap B$, there exists $\lambda_{1 x} \in \Lambda_{1}, \lambda_{2 x} \in \Lambda_{2}$ such that $x \in B_{\lambda_{1 x}} \cap B_{\lambda_{2 x}}$. From the assumption, we know that

$$
t<\beta_{P}\left(B_{\lambda_{1 x}}\right) \wedge \beta_{P}\left(B_{\lambda_{2 x}}\right) \leq \bigvee_{x \in C \subseteq B_{\lambda_{1 x}} \cap B_{\lambda_{2 x}}} \beta_{P}(C),
$$

and furthermore, there exists $C_{x}$ such that

$$
x \in C_{x} \subseteq B_{\lambda_{1 x}} \cap B_{\lambda_{2 x}} \subseteq A \cap B, \quad \beta_{P}\left(C_{x}\right)>t .
$$

Since

$$
\bigcup_{x \in A \cap B} C_{x}=A \cap B
$$

we have

$$
t \leq \bigwedge_{x \in A \cap B} \beta_{P}\left(C_{x}\right) \leq \bigcup_{\lambda \in \Lambda} \bigvee_{\lambda} \bigwedge_{\lambda=A \cap B} \beta_{\lambda \in \Lambda}\left(B_{\lambda}\right)=\tau_{P}(A \cap B) .
$$

Now, let $\tau_{P}(A) \wedge \tau_{P}(B)=k$. For any natural number $n$, we have

$$
\tau_{P}(A)>k-\frac{1}{n}, \quad \tau_{P}(B)>k-\frac{1}{n}
$$

and so $\tau_{P}(A \cap B) \geq k-\frac{1}{n}$. Therefore $\tau_{P}(A \cap B) \geq k=\tau_{P}(A) \wedge \tau_{P}(B)$.

Definition 2.2. $\varphi_{P}: 2^{X} \rightarrow[0,1]$ is called a pre-subbase of $\tau_{P}$ if $\varphi_{P}^{(\cap)}$ is a pre-base of $\tau_{P}$, where

$$
\varphi_{P}^{(\cap)}\left(\bigcap_{\lambda \in \Lambda} B_{\lambda}\right)=\bigcap_{\lambda \in \Lambda} B_{\lambda}=A \bigwedge_{\lambda \in \Lambda} \varphi_{P}\left(B_{\lambda}\right),
$$

$\left\{B_{\lambda}: \lambda \in \Lambda\right\} \sqsubset P(X)$ with " $\sqsubset "$ standing for "a finite subset of".

Theorem 2.3. $\varphi_{P}: 2^{X} \rightarrow[0,1]$ is a pre-subbase of some fuzzifying $P$ topology if and only if $\varphi_{P}^{(\cup)}(X)=1$. 
Proof. We only demonstrate that $\varphi_{P}^{(\cap)}$ satisfies the second condition of Theorem 2.2, and others are obvious. In fact

$$
\begin{aligned}
& \varphi_{P}^{(\cap)}(A) \wedge \varphi_{P}^{(\cap)}(B) \\
& =\left(\bigcap_{\lambda_{1} \in \Lambda_{1}} \bigvee_{B_{\lambda_{1}}=A} \bigwedge_{\lambda_{1} \in \Lambda_{1}} \varphi_{P}\left(B_{\lambda_{1}}\right)\right) \wedge\left(\bigwedge_{\bigcap_{\lambda_{2} \in \Lambda_{2}} B_{\lambda_{2}}=B^{\lambda_{2} \in \Lambda_{2}}} \bigwedge_{P}\left(B_{\lambda_{2}}\right)\right) \\
& =\bigvee_{\bigcap_{1} \in \Lambda_{1}} \bigvee_{B_{\lambda_{1}}=A} \bigcap_{\bigcap_{\lambda_{2} \in \Lambda_{2}} B_{\lambda_{2}}=B}\left(\bigwedge_{\lambda_{1} \in \Lambda_{1}} \varphi_{P}\left(B_{\lambda_{1}}\right)\right) \wedge\left(\bigwedge_{\lambda_{2} \in \Lambda_{2}} \varphi_{P}\left(B_{\lambda_{2}}\right)\right) \\
& \leq \bigvee_{\substack{\lambda \in \Lambda \\
B_{\lambda}=A \cap B}}\left(\bigwedge_{\lambda \in \Lambda} \varphi_{P}\left(B_{\lambda}\right)\right) \\
& =\varphi_{P}^{(\cap)}(A \cap B) .
\end{aligned}
$$

Therefore, if $x \in A \cap B$, then

$$
\varphi_{P}^{(\cap)}(A) \wedge \varphi_{P}^{(\cap)}(B) \leq \varphi_{P}^{(\cap)}(A \cap B) \leq \bigvee_{x \in C \subseteq A \cap B} \varphi_{P}^{(\cap)}(C)
$$

\section{Fuzzifying strong compact spaces}

Theorem 3.1. Let $(X, \tau)$ be a fuzzifying topological space, $\varphi_{P}$ be a presubbase of $\tau_{P}$, and

$\left(\beta_{1}\right):=(\forall \Re)\left(K_{\varphi_{P}}(\Re, X) \rightarrow \exists \wp((\wp \leq \Re) \wedge K(\wp, X) \otimes F F(\wp))\right)$, where $K_{\varphi_{P}}(\Re, X):=K(\Re, X) \otimes\left(\Re \subseteq \varphi_{P}\right)$;

$\left(\beta_{2}\right):=(\forall S)\left((S\right.$ is a universal net in $\left.X) \rightarrow \exists x\left((x \in X) \wedge\left(S \triangleright^{P} x\right)\right)\right)$;

$\left(\beta_{3}\right):=(\forall S)\left(\left(S \in N(X) \rightarrow(\exists T)(\exists x)\left((T<S) \wedge(x \in X) \wedge\left(T \triangleright^{P} x\right)\right)\right)\right.$,

where "T<S" stands for " $T$ is a subnet of $S "$;

$\left(\beta_{4}\right):=(\forall S)\left(\left(S \in N(X) \rightarrow \neg\left(a d h_{P} S \equiv \phi\right)\right) ;\right.$

$\left(\beta_{5}\right):=(\forall \Re)\left(\Re \in[0,1]^{2^{X}} \wedge \Re \subseteq \mathcal{F}_{P} \otimes f I(\Re) \rightarrow \exists x \forall A(A \in \Re \rightarrow x \in A)\right)$.

Then $\models(X, \tau) \in \Gamma_{P} \leftrightarrow \beta_{i} i=1,2, \ldots, 5$. 
Proof. (1) Since $\varphi_{P} \subseteq \tau_{P},\left[\Re \subseteq \varphi_{P}\right] \leq\left[\Re \subseteq \tau_{P}\right]$ for any $\Re: 2^{X} \rightarrow[0,1]$, then we have $\left[K_{\varphi_{P}}(\Re, X)\right] \leq\left[K_{P}(\Re, X)\right]$. Therefore $\Gamma_{P}(X, \tau) \leq\left[\beta_{1}\right]$.

(2) $\left[\beta_{2}\right]=\bigwedge\left\{\bigvee_{x \in X}\left[S \triangleright^{P} x\right]:(S\right.$ is a universal net in $\left.X)\right\}$.

(2.1) Assume $X$ is finite. We set $X=\left\{x_{1}, \ldots, x_{m}\right\}$. For any universal net $S$ in $X$, there exists $i_{\circ} \in\{1, \ldots, m\}$ with $S \widetilde{\subset}\left\{x_{i_{\circ}}\right\}$. In fact, if not, then for any $i \in\{1, \ldots, m\}, S \widetilde{\not \subset}\left\{x_{i_{\circ}}\right\}, S \widetilde{\subset} X-\left\{x_{i_{\circ}}\right\}$ and $S \widetilde{\subset} \bigcap_{i=1}^{m}\left(X-\left\{x_{i}\right\}\right)=\phi$, a contradiction. Therefore $x_{i_{\circ}} \notin A$ and $N_{x_{i_{\circ}}}^{P}(A)=0$ (see[2], Theorem 4.2

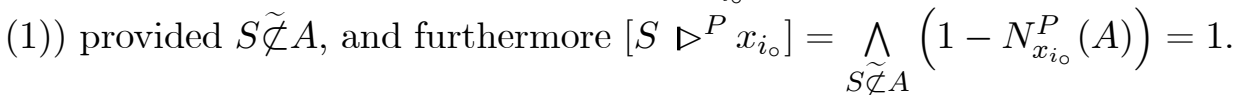
Therefore $\left[\beta_{2}\right]=1 \geq\left[\beta_{1}\right]$.

(2.2) In general, to prove that $\left[\beta_{1}\right] \leq\left[\beta_{2}\right]$ we prove that for any $\lambda \in[0,1]$, if $\left[\beta_{2}\right]<\lambda$, then $\left[\beta_{1}\right]<\lambda$. Assume for any $\lambda \in[0,1],\left[\beta_{2}\right]<\lambda$. Then, there exists a universal net $S$ in $X$ such that $\bigvee_{x \in X}\left[S \triangleright^{P} x\right]<\lambda$ and for any $x \in X,\left[S \triangleright^{P} x\right]=\bigwedge_{S \widetilde{\not \subset A}}\left(1-N_{x}^{P}(A)\right)<\lambda$, i.e., there exists $A \subseteq X$ with $S \widetilde{\not \subset} A$ and $N_{x}^{P}(A)>1-\lambda$. Since $\varphi_{P}$ is a pre-subbase of $\tau_{P}, \varphi_{P}^{(\cap)}$ is a pre-base of $\tau_{P}$ and from Definition 2.1, we have $\bigvee_{x \in B \subseteq A} \varphi_{P}^{(\cap)}(B) \geq$ $N_{x}^{P}(A)>1-\lambda$, i.e., there exists $B \subseteq A$ such that $x \in B \subseteq A$ and $\bigvee\left\{\min _{\lambda \in \Lambda} \varphi_{P}\left(B_{\lambda}\right): \bigcap_{\lambda \in \Lambda} B_{\lambda}=B, B_{\lambda} \subseteq X, \lambda \in \Lambda\right\}=\varphi_{P}^{(\cap)}(B)>1-\lambda$, where $\Lambda$ is finite. Therefore, there exists a finite set $\Lambda$ and $B_{\lambda} \subseteq X(\lambda \in \Lambda)$ such that $\bigcap_{\lambda \in \Lambda} B_{\lambda}=B$ and for any $\lambda \in \Lambda, \varphi_{P}\left(B_{\lambda}\right)>1-\lambda$. Since $S \widetilde{\not \subset} A$ and $\Lambda$ is finite, there exists $\lambda(x) \in \Lambda$ such that $S \widetilde{\not \subset} B_{\lambda(x)}$. We set

$$
\Re_{\circ}\left(B_{\lambda(x)}\right)=\bigvee_{x \in X} \varphi_{P}\left(B_{\lambda(x)}\right) .
$$

If $\wp \leq \Re_{\circ}$, then for any $\delta>0, \wp_{\delta} \subseteq\left\{B_{\lambda(x)}: x \in X\right\}$. Consequently, for any $B \in \wp_{\delta}, S \widetilde{\not \subset B}$ and $S \widetilde{\subset} B^{c}$ because $S$ is a universal net. If

$$
[F F(\wp)]=1-\inf \left\{\delta \in[0,1]: F\left(\wp_{\delta}\right)\right\}=t,
$$

then for any $n \in w$ (the non-negative integer),

$$
\inf \left\{\delta \in[0,1]: F\left(\wp_{\delta}\right)\right\}<1-t+\frac{1}{n},
$$

and there exists $\delta_{\circ}<1-t+\frac{1}{n}$ such that $F\left(\wp_{\delta}\right)$. If $\delta_{\circ}=0$, then $P(X)=\wp_{\delta} \circ$ is finite and it is proved in (2.1). If $\delta_{\circ}>0$, then for any $B \in \wp_{\delta_{\circ}}, S \widetilde{\subset} B^{c}$. 
Since $F\left(\wp_{\delta}\right)$, we have

$$
S \widetilde{\subset} \bigcap\left\{B^{c}: B \in \wp_{\delta \circ}\right\} \neq \phi .
$$

i.e., $\bigcup \wp_{\circ} \neq X$ and there exist $x_{\circ} \in X$ such that for any $B \in \wp_{\delta}, x_{\circ} \notin B$. Therefore, if $x_{\circ} \in B$, then $B \notin \wp \delta$, i.e., $\wp(B)<\delta \circ$,

$$
K(\wp, X)=\bigwedge_{x \in X} \bigvee_{x \in B} \wp(B) \leq \bigvee_{x_{\circ} \in B} \wp(B) \leq \delta \circ<1-t+\frac{1}{n} .
$$

Let $n \rightarrow \infty$. We obtain $K(\wp, X) \leq 1-t$ and $[K(\wp, X) \otimes F F(\wp)]=0$. In addition, $\left[K_{\varphi_{P}}\left(\Re_{\circ}, X\right)\right] \geq 1-\lambda$. In fact, $\left[\Re_{\circ} \subseteq \varphi_{P}\right]=1$ and

$$
\left.K\left(\Re_{\circ}, X\right)\right]=\bigwedge_{x \in X} \bigvee_{x \in B} \Re_{\circ}(B) \geq \bigwedge_{x \in X} \Re_{\circ}\left(B_{\lambda(x)}\right) \geq \bigwedge_{x \in X} \varphi_{P}\left(B_{\lambda(x)}\right) \geq 1-\lambda
$$

because $x \in B_{\lambda(x)}$. Now, we have

$$
\begin{aligned}
{\left[\beta_{1}\right] } & =\left[(\forall \Re)\left(K_{\varphi_{P}}(\Re, X) \rightarrow \exists \wp((\wp \leq \Re) \wedge K(\wp, X) \otimes F F(\wp))\right)\right] \\
& \leq\left[K_{\varphi_{P}}\left(\Re_{\circ}, X\right) \rightarrow \exists \wp((\wp \leq \Re \circ) \wedge K(\wp, X) \otimes F F(\wp))\right] \\
& =\min \left(1,1-K_{\varphi_{P}}\left(\Re_{\circ}, X\right)+\bigvee_{\wp \leq \Re_{\circ}}[K(\wp, X) \otimes F F(\wp))\right] \leq \lambda .
\end{aligned}
$$

Since $\lambda$ is arbitrary, we have $\left[\beta_{1}\right] \leq\left[\beta_{2}\right]$.

(3) It is immediate that $\left[\beta_{2}\right] \leq\left[\beta_{3}\right]$.

(4) To prove that $\left[\beta_{3}\right] \leq\left[\beta_{4}\right]$, first we prove that

$$
\left[\exists T\left((T<S) \wedge\left(T \triangleright^{P} x\right)\right)\right] \leq\left[S \propto^{P} x\right],
$$

where

$$
\left[\exists T\left((T<S) \wedge\left(T \triangleright^{P} x\right)\right)\right]=\bigvee_{T<S} \bigwedge_{T \widetilde{\not \subset A}}\left(1-N_{x}^{P}(A)\right)
$$

and

$$
\left[S \propto^{P} x\right]=\bigwedge_{S \widetilde{\not \subset A} A}\left(1-N_{x}^{P}(A)\right) .
$$

Indeed, for any $T<S$ one can deduce $\{A: S \widetilde{\not} A\} \subseteq\{A: T \widetilde{\not \subset} A\}$ as follows. Suppose $T=S \circ K$. If $S \widetilde{\not} A$, then there exists $m \in D$ such that $S(n) \notin A$ when $n \geq m$, where $\geq$ directs the domain $D$ of $S$. Now, we will show that $T \tilde{\not \subset} A$. If not, then there exists $p \in E$ such that $T(q) \in A$ when $q \geq p$, where $\geq$ directs the domain $E$ of $T$. Moreover, there exists $n_{1} \in E$ such that 
$K\left(n_{1}\right) \geq m$ because $T<S$, and there exists $n_{2} \in E$ such that $n_{2} \geq n_{1}, p$ because $(E, \geq)$ is directed. So, $K\left(n_{2}\right) \geq K\left(n_{1}\right) \geq m, S \circ K\left(n_{2}\right) \notin A$ and $S \circ K\left(n_{2}\right)=T\left(n_{2}\right) \in A$. They are contrary. Hence,

$$
\{A: S \widetilde{\not \subset} A\} \subseteq\{A: T \widetilde{\not \subset} A\} .
$$

Therefore,

$$
\begin{aligned}
{\left[\exists T\left((T<S) \wedge\left(T \triangleright^{P} x\right)\right)\right] } & =\bigvee_{T<S} \bigwedge_{T \widetilde{\not} A}\left(1-N_{x}^{P}(A)\right) \\
& =\bigvee_{T<S} \bigwedge_{\{A: \widetilde{T \subset} A\}}\left(1-N_{x}^{P}(A)\right) \\
& \leq \bigwedge_{\{A: S \widetilde{\not \subset} A\}}\left(1-N_{x}^{P}(A)\right) \\
& =\bigwedge_{S \widetilde{\not \subset} A}\left(1-N_{x}^{P}(A)\right)=\left[S \alpha^{P} x\right]
\end{aligned}
$$

Therefore, for any $x \in X$ and $S \in N(X)$ we have

$$
\begin{aligned}
{\left[\beta_{3}\right] } & =\bigwedge_{S \in N(X)} \bigvee_{x \in X}\left[\exists T\left((T<S) \wedge\left(T \triangleright^{P} x\right)\right)\right] \\
& \leq \bigwedge_{S \in N(X)} \bigvee_{x \in X}\left[S \propto^{P} x\right]=\bigwedge_{S \in N(X)} \neg\left(\bigwedge_{x \in X}\left(1-\left[S \propto^{P} x\right]\right)\right) \\
& =\bigwedge_{S \in N(X)}\left[\neg\left(a d h_{P} S \equiv \phi\right)\right]=\left[\beta_{4}\right] .
\end{aligned}
$$

(5) We want to show that $\left[\beta_{4}\right] \leq\left[\beta_{5}\right]$. For any $\Re: 2^{X} \rightarrow[0,1]$, assume $[f I(\Re)]=\lambda$. Then for any $\delta>1-\lambda$, if $A_{1}, \ldots, A_{n} \in \Re_{\delta}, A_{1} \cap A_{2} \cap \ldots \cap A_{n} \neq \phi$. In fact, we set $\wp\left(A_{i}\right)=\bigvee_{i=1}^{n} \Re\left(A_{i}\right)$. Then $\wp \leq \Re$ and $F F(\wp)=1$. By putting $\varepsilon=\lambda+\delta-1>0$, we obtain

$$
\lambda-\varepsilon<\lambda \leq[F F(\wp) \rightarrow(\exists x)(\forall B)(B \in \wp \rightarrow x \in B)]=\bigvee_{x \in X} \bigwedge_{x \notin B}(1-\wp(B))
$$

There exists $x_{\circ} \in X$ such that $\lambda-\varepsilon<\bigwedge_{x_{\circ} \notin B}(1-\wp(B)), x_{\circ} \notin B$ implies $\wp(B)<1-\lambda+\varepsilon=\delta$ and $x_{\circ} \in \cap \wp_{\delta}=A_{1} \cap A_{2} \cap \ldots \cap A_{n}$. Now, we set $\vartheta_{\delta}=\left\{A_{1} \cap A_{2} \cap \ldots \cap A_{n}: n \in N, A_{1}, \ldots, A_{n} \in \Re_{\delta}\right\}$ and $S: \vartheta_{\delta} \rightarrow X, B \mapsto$ 
$x_{B} \in B, B \in \vartheta_{\delta}$ and know that $\left(\vartheta_{\delta}, \subseteq\right)$ is a directed set and $S$ is a net in $X$. Therefore,

$$
\left[\beta_{4}\right] \leq\left[\neg\left(a d h_{P} S \equiv \phi\right)\right]=\bigvee_{x \in X} \bigwedge_{S \widetilde{\not \subset A} A}\left(1-N_{x}^{P}(A)\right) .
$$

Assume that $\left[\Re \subseteq F_{P}\right]=\mu$. Then, for any $B \in P(X), \Re(B) \leq 1+F_{P}(B)-\mu$, and

$$
\begin{aligned}
{[\Re} & \left.\subseteq F_{P} \otimes f I(A) \rightarrow(\exists x)(\forall A)((A \in \Re) \rightarrow x \in A)\right] \\
& =\min \left(1,2-\mu-\lambda+\bigvee_{x \in X} \bigwedge_{x \notin A}(1-\Re(A))\right) .
\end{aligned}
$$

Therefore, it suffices to show that for any $\mathrm{x} \in X$,

$$
\bigwedge_{S \widetilde{\not} A}\left(1-N_{x}^{P}(A)\right) \leq 2-\mu-\lambda+\bigwedge_{x \notin A}(1-\Re(A)),
$$

i.e.,

$$
\bigvee_{x \notin A} \Re(A) \leq 2-\mu-\lambda+\bigvee_{S \widetilde{\not Z A}} N_{x}^{P}(A)
$$

for some $\delta>1-\lambda$.For any $t \in[0,1]$, if $\underset{x \notin A}{\bigvee} \Re(A)>t$, then there exists $A_{\circ}$ such that $x_{\circ} \notin A_{\circ}$ and $\Re\left(A_{\circ}\right)>t$.

Case 1. $t \leq 1-\lambda$, then $t \leq 2-\mu-\lambda+\underset{S \widetilde{\not \subset A}}{\bigvee} N_{x}^{P}(A)$

Case 2. $t>1-\lambda$. Here we set $\delta=\frac{1}{2}(t+1-\lambda)$ and have $A_{\circ} \in \Re_{\delta}, A_{\circ} \in \vartheta_{\delta}$. In addition,

$$
t<\Re\left(A_{\circ}\right) \leq 1+F_{P}\left(A_{\circ}\right)-\mu, t+\mu-1 \leq F_{P}\left(A_{\circ}\right)=\tau_{P}\left(A_{\circ}^{c}\right) .
$$

Since $A_{\circ} \in \vartheta_{\delta}$, we know that $S_{B} \in A_{\circ}$, i.e., $S_{B} \notin A_{\circ}^{c}$ when $B \subseteq A_{\circ}$ and $S \widetilde{\not} A_{\circ}^{c}$. Therefore,

$$
2-\mu-\lambda+\bigvee_{S \widetilde{\not} A} N_{x}^{P}(A) \geq 2-\mu-\lambda+N_{x}^{P}\left(A_{\circ}^{c}\right) \geq 2-\mu-\lambda+\tau_{P}\left(A_{\circ}^{c}\right) \geq t+(1-\lambda) \geq t .
$$

By noticing that $t$ is arbitrary, we have completed the proof.

(6) To prove that $\left[\beta_{5}\right]=\left[(X, \tau) \in \Gamma_{P}\right][1$, Theorem 6$]$.

The above theorem is a generalization of the following corollary. 
Corollary 3.1. The following are equivalent for a topological space $(X, \tau)$.

(a) $X$ is a strong compact space.

(b) Every cover of $X$ by members of a pre-subbase of $\tau_{P}$ has a finite subcover.

(c) Every universal net in $X$ pre-converges to a point in $X$.

(d) Each net in $X$ has a subnet that pre-converges to some point in $X$.

(e) Each net in $X$ has a pre-adherent point.

(f) Each family of pre-closed sets in $X$ that has the finite intersection property has a non-void intersection.

The equivalence (a), (b) and (c) were given in [16].

Definition 3.1. Let $\left\{\left(X_{s}, \tau_{s}\right): s \in S\right\}$ be a family of fuzzifying topological spaces, $\prod_{s \in S} X_{s}$ be the cartesian product of $\left\{X_{s}: s \in S\right\}$ and $\varphi=\left\{p_{s}^{-1}\left(U_{s}\right)\right.$ : $\left.s \in S, U_{s} \in P\left(X_{s}\right)\right\}$, where $p_{t}: \prod_{s \in S} X_{s} \rightarrow X_{t}(t \in S)$ is a projection. For $\Phi \subseteq \varphi, S(\Phi)$ stands for the set of indices of elements in $\Phi$. The pre-base $\beta_{P}: \prod_{s \in S} X_{s} \rightarrow[0,1]$ of $\prod_{s \in S}\left(\tau_{P}\right)_{s}$ is defined as

$$
V \in \beta_{P}:=(\exists \Phi)(\Phi \sqsubset \varphi \wedge(\bigcap \Phi=V)) \rightarrow \forall s\left(s \in S(\Phi) \rightarrow V_{s} \in\left(\tau_{P}\right)_{s}\right),
$$
i.e.,

$$
\beta_{P}(V)=\bigvee_{\Phi \sqsubset \varphi, \bigcap \Phi=V} \bigwedge_{s \in S(\Phi)}\left(\tau_{P}\right)_{s}\left(V_{s}\right) .
$$

Example 3.1. Let $(X, \tau)$ and $\tau_{P}$ be just as in Example 2.1. Define a mapping $\varsigma: P(Y) \longrightarrow I$ on $Y$ as follows: $\varsigma(\emptyset)=\varsigma(Y)=1$, where $Y=\{d\}$, then $\varsigma$ is a fuzzifying topology and $\varsigma_{P}(\emptyset)=\varsigma_{P}(Y)=1$. Hence, $Y \times X=\{(d, a),(d, b),(d, c)\}$, so $\varphi=\{\emptyset, X \times Y,\{(d, a)\},\{(d, b)\},\{(d, c)\}$, $\{(d, a),(d, b)\},\{(d, b),(d, c)\},\{(d, a),(d, c)\}\}$. By calculating,

$\beta_{p}(\emptyset)=1, \beta_{P}(X \times Y)=1, \beta_{P}(\{(d, a)\})=\frac{1}{4}, \beta_{P}(\{(d, b)\})=\frac{3}{4}, \beta_{P}(\{(d, c)\})=$ $\frac{1}{2}, \beta_{P}(\{(d, a),(d, c)\})=\frac{1}{4}, \beta_{P}(\{(d, a),(d, b)\})=\frac{1}{2}, \beta_{P}(\{(d, b),(d, c)\})=1$.

According to Theorem 2.1, we can easily obtain $\beta_{P}^{(\cup)}=\beta_{P}$, so $\tau_{P} \times \varsigma_{P}=\beta_{P}$.

Definition 3.2. Let $(X, \tau),(Y, \sigma)$ be two fuzzifying topological space. A unary fuzzy predicate $O_{P}: Y^{X} \rightarrow[0,1]$, is called fuzzifying pre-openness, is given as: $O_{P}(f):=\forall U\left(U \in \tau_{P} \rightarrow f(U) \in \sigma_{P}\right)$. Intuitively, the degree to which $f$ is pre-open is

$$
\left[O_{P}(f)\right]=\bigwedge_{B \subseteq X} \min \left(1,1-\tau_{P}(U)+\sigma_{P}(f(U))\right) .
$$


Example 3.2. Let $(X, \tau)$ and $\tau_{P}$ be defined just as in Example 2.1. We set $Y=X, \sigma=\tau$ and $f=i d_{X}$, then $\left[O_{P}(f)\right]=\bigwedge_{U \subseteq X} \min \left(1,1-\tau_{P}(U)+\right.$ $\left.\sigma_{P}(f(U))\right)=1$.

Lemma 3.1. Let $(X, \tau)$ and $(Y, \sigma)$ be two fuzzifying topological space. For any $f \in Y^{X}$,

$$
O_{P}(f):=\forall B\left(B \in \beta_{P}^{X} \rightarrow f(B) \in \sigma_{P}\right),
$$

where $\beta_{P}^{X}$ is a pre-base of $\tau_{P}$.

Proof. Clearly, $\left[O_{P}(f)\right] \leq\left[\forall U\left(U \in \beta_{P}^{X} \rightarrow f(U) \in \sigma_{P}\right)\right]$. Conversely, for any $U \subseteq X$, we are going to prove

$$
\min \left(1,1-\tau_{P}(U)+\sigma_{P}(f(U))\right) \geq\left[\forall V\left(V \in \beta_{P}^{X} \rightarrow f(V) \in \sigma_{P}\right)\right] .
$$

If $\tau_{P}(U) \leq \sigma_{P}(f(U))$, it is hold clearly. Now, assume $\tau_{P}(U)>\sigma_{P}(f(U))$. If $\Re \subseteq P(X)$ with $\bigcup \Re=U$, then $\bigcup_{V \in \Re} f(V)=f(\bigcup \Re)=f(U)$. Therefore,

$$
\begin{aligned}
& \tau_{\mathrm{P}}(\mathrm{U})-\sigma_{P}(f(U))
\end{aligned}
$$

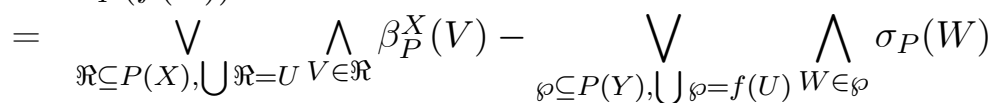

$$
\begin{aligned}
& \leq \bigvee_{\Re \subseteq P(X), \bigcup \Re=U} \bigwedge_{V \in \Re} \beta_{P}^{X}(V)-\bigvee_{\Re \subseteq P(X), \bigcup \Re=U} \bigwedge_{V \in \Re} \sigma_{P}(f(V))
\end{aligned}
$$

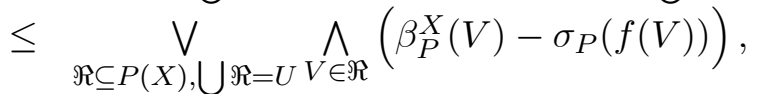

$$
\begin{aligned}
& \min \left(1,1-\tau_{P}(U)+\sigma_{P}(f(U))\right) \\
& \geq \underset{\Re \subseteq P(X), \bigcup \Re=U}{\bigvee} \bigwedge_{V \in \Re} \min \left(1,1-\beta_{P}^{X}(V)+\sigma_{P}(f(V))\right) \\
& \geq\left[\forall V\left(V \in \beta_{P}^{X} \rightarrow f(V) \in \sigma_{P}\right)\right] .
\end{aligned}
$$

Lemma 3.2. For any family $\left\{\left(X_{s}, \tau_{s}\right): s \in S\right\}$ of fuzzifying topological spaces.
(1) $=(\forall s)\left(s \in S \rightarrow p_{s} \in O_{P}\right)$;
$(2) \models(\forall s)\left(s \in S \rightarrow p_{s} \in C_{P}\right)$.

Proof. (1) For any $t \in S$, we have

$$
O_{P}\left(p_{t}\right)=\bigwedge_{U \in P\left(\prod_{s \in S} X_{s}\right)} \min \left(1,1-\left(\prod_{s \in S}\left(\tau_{P}\right)_{s}\right)(U)+\left(\tau_{P}\right)_{t}\left(p_{t}(U)\right)\right) .
$$


Then, it suffices to show that for any $U \in P\left(\prod_{s \in S} X_{s}\right)$, we have

$$
\left(\tau_{P}\right)_{t}\left(p_{t}(U)\right) \geq\left(\prod_{s \in S}\left(\tau_{P}\right)_{s}\right)(U)
$$

Assume

$$
\left(\prod_{s \in S}\left(\tau_{P}\right)_{s}\right)(U)=\bigvee_{\substack{u \\ B_{\lambda}=U \\ \lambda \in \Lambda}} \bigwedge_{\lambda \in \Lambda} \bigvee_{\Phi_{\lambda} \sqsubset \varphi, \cap \Phi_{\lambda}=B_{\lambda}} \bigwedge_{s \in S\left(\Phi_{\lambda}\right)}\left(\tau_{P}\right)_{s}\left(V_{s}\right)>\mu
$$

where

$$
\Phi_{\lambda}=\left\{p_{s}^{-1}\left(V_{s}\right): s \in S\left(\Phi_{\lambda}\right)\right\}(\lambda \in \Lambda) .
$$

Hence, there exists $\left\{B_{\lambda}: \lambda \in \Lambda\right\} \subseteq P\left(\prod_{s \in S} X_{s}\right)$ such that $\bigcup_{\lambda \in \Lambda} B_{\lambda}=U$ and furthermore, for any $\lambda \in \Lambda$, there exists $\Phi_{\lambda} \sqsubset \varphi$ such that $\cap \Phi_{\lambda}=B_{\lambda}$ and $\bigcap_{s \in S\left(\Phi_{\lambda}\right)} p_{s}^{-1}\left(V_{s}\right)=B_{\lambda}$, where for any $s \in S\left(\Phi_{\lambda}\right)$ we have $\left(\tau_{P}\right)_{s}\left(V_{s}\right)>\mu$. Thus,

$$
p_{t}(U)=p_{t}\left(\bigcup_{\lambda \in \Lambda} \bigcap_{s \in S\left(\Phi_{\lambda}\right)} p_{s}^{-1}\left(V_{s}\right)\right) .
$$

(1) If for any $\lambda \in \Lambda, \bigcap_{s \in S\left(\Phi_{\lambda}\right)} p_{s}^{-1}\left(V_{s}\right)=\phi$, then $U=\phi, p_{t}(U)=\phi$ and $\left(\tau_{P}\right)_{t}\left(p_{t}(U)\right)=1$. Therefore, $\left(\tau_{P}\right)_{t}\left(p_{t}(U)\right) \geq\left(\prod_{s \in S}\left(\tau_{P}\right)_{s}\right)(U)$.

(2) If there exists $\lambda_{\circ} \in \Lambda$, such that $\phi \neq \bigcap_{s \in S\left(\Phi_{\lambda}\right)} p_{s}^{-1}\left(V_{s}\right)=B_{\lambda_{\circ}}$,

(i) If $t \notin S\left(\Phi_{\lambda_{\circ}}\right)$, i.e., $t \in S-S\left(\Phi_{\lambda_{\circ}}\right), p_{t}\left(B_{\lambda_{\circ}}\right)=X_{t}$. Therefore, $\left(\tau_{P}\right)_{t}\left(p_{t}\left(B_{\lambda_{\circ}}\right)\right)=\left(\tau_{P}\right)_{t}\left(X_{t}\right)=1$.

(ii) If $t \in S\left(\Phi_{\lambda_{\circ}}\right)$, then $p_{t}\left(B_{\lambda_{\circ}}\right)=V_{t} \subseteq X_{t}$. Thus,

$$
\begin{gathered}
p_{t}(U)=p_{t}\left(\left(\bigcup_{t \in S\left(\Phi_{\lambda_{\diamond}}\right)} B_{\lambda_{\circ}}\right) \cup\left(\bigcup_{t \notin S\left(\Phi_{\lambda_{\circ}}\right)} B_{\lambda_{\circ}}\right)\right) \\
=\left(\bigcup_{t \in S\left(\Phi_{\lambda_{\circ}}\right)} p_{t}\left(B_{\lambda_{\circ}}\right)\right) \cup\left(\bigcup_{t \notin S\left(\Phi_{\lambda_{\circ}}\right)} p_{t}\left(B_{\lambda_{\diamond}}\right)\right)=V_{t} \cup X_{t}=X_{t} .
\end{gathered}
$$

Hence,

$$
\left(\tau_{P}\right)_{t}\left(p_{t}(U)\right)=\left(\tau_{P}\right)_{t}\left(X_{t}\right)=1 \text { or }\left(\tau_{P}\right)_{t}\left(p_{t}(U)\right)=\left(\tau_{P}\right)_{t}\left(V_{t}\right)>\lambda .
$$


Therefore, $\left(\tau_{P}\right)_{t}\left(p_{t}(U)\right) \geq\left(\prod_{s \in S}\left(\tau_{P}\right)_{s}\right)(U)$. Thus $O_{P}\left(p_{t}\right)=1$.

(2) From Lemma 3.1 in [32], we have $=(\forall s)\left(s \in S \rightarrow p_{s} \in C\right)$. Furthermore, for any two fuzzifying topological spaces $(X, \tau)$ and $(Y, \sigma)$ and $f \in Y^{X}$, we have $C(f) \leq C_{P}(f)$ (Theorem 6.3 in [2]). Therefore, $\models(\forall s)\left(s \in S \rightarrow p_{s} \in C_{P}\right)$.

Theorem 3.2. Let $\left\{\left(X_{s}, \tau_{s}\right): s \in S\right\}$ be the family of fuzzifying topological spaces, then

$$
\begin{gathered}
\models \exists U\left(U \subseteq \prod_{s \in S} X_{s} \wedge \Gamma_{P}(U, \tau / U) \wedge \exists x\left(x \in \operatorname{Int}_{P}(U)\right)\right. \\
\rightarrow \exists T\left(T \sqsubset S \wedge \forall t\left(t \in S-T \wedge \Gamma_{P}\left(X_{t}, \tau_{t}\right)\right)\right) .
\end{gathered}
$$

Proof. It suffices to show that

$$
\begin{gathered}
\bigvee_{U \in P\left(\prod_{s \in S} X_{s}\right)}\left(\Gamma_{P}(U, \tau / U) \wedge \bigvee_{x \in \prod_{s \in S} X_{s}} N_{x}^{P}(U)\right) \\
\leq \bigvee_{T \sqsubset S} \bigwedge_{t \in S-T} \Gamma_{P}\left(X_{t}, \tau_{t}\right) .
\end{gathered}
$$

Indeed, if

$$
\bigvee_{U \in P\left(\prod_{s \in S} X_{s}\right)}\left(\Gamma_{P}(U, \tau / U) \wedge \bigvee_{x \in \prod_{s \in S} X_{s}} N_{x}^{P}(U)\right)>\mu>0,
$$

then there exists $U \in P\left(\prod_{s \in S} X_{s}\right)$ such that $\Gamma_{P}(U, \tau / U)>\mu$ and $\bigvee_{x \in \prod_{s \in S} X_{s}} N_{x}^{P}(U)>\mu$, where

$$
N_{x}^{P}(U)=\bigvee_{x \in V \subseteq U}\left(\prod_{s \in S}\left(\tau_{P}\right)_{s}\right)(V)
$$


Furthermore, there exists $V$ such that $x \in V \subseteq U$ and $\left(\prod_{s \in S}\left(\tau_{P}\right)_{s}\right)(V)>$ $\mu$. Since $\beta_{P}$ is a pre-base of $\prod_{s \in S}\left(\tau_{P}\right)_{s}$,

$$
\begin{aligned}
\left(\prod_{s \in S}\left(\tau_{P}\right)_{s}\right)(V)= & \bigvee_{\lambda \in \Lambda} \bigwedge_{\lambda \in \Lambda} \beta_{P}\left(B_{\lambda}\right) \\
= & \bigcup_{\lambda \in \Lambda} B_{\lambda}=V \bigwedge_{\lambda \in \Lambda} \bigvee_{\Phi_{\lambda} \sqsubset \varphi, \cap \Phi_{\lambda}=B_{\lambda}} \bigwedge_{s \in S\left(\Phi_{\lambda}\right)}\left(\tau_{P}\right)_{s}\left(V_{s}\right)>\mu,
\end{aligned}
$$

where $\Phi_{\lambda}=\left\{p_{s}^{-1}\left(V_{s}\right): s \in S\left(\Phi_{\lambda}\right)\right\}(\lambda \in \Lambda)$.

Hence there exists $\left\{B_{\lambda}: \lambda \in \Lambda\right\} \subseteq P\left(\prod_{s \in S} X_{s}\right)$ such that $\cup_{\lambda \in \Lambda} B_{\lambda}=V$.

Furthermore, for any $\lambda \in \Lambda$, there exists $\Phi_{\lambda} \sqsubset \varphi$ such that $\cap \Phi_{\lambda}=B_{\lambda}$ and for any $s \in S\left(\Phi_{\lambda}\right)$, we have $\left(\tau_{P}\right)_{s}\left(V_{s}\right)>\mu$. Since $x \in V$, there exists $B_{\lambda_{x}}$ such that $x \in B_{\lambda_{x}} \subseteq V \subseteq U$. Hence there exists $\Phi_{\lambda_{x}} \sqsubset \varphi$ such that $\cap \Phi_{\lambda_{x}}=B_{\lambda_{x}}$ and $\bigcap_{s \in S\left(\Phi_{\lambda}\right)} p_{s}^{-1}\left(V_{s}\right)=B_{\lambda_{x}} \subseteq \prod_{s \in S} X_{s}$ and for any $s \in S\left(\Phi_{\lambda}\right)$, we have $\left(\tau_{P}\right)_{s}\left(V_{s}\right)>1-\mu$. By $\bigcap_{s \in S\left(\Phi_{\lambda}\right)} p_{s}^{-1}\left(V_{s}\right)=B_{\lambda_{x}}$, we have $P_{\delta}\left(B_{\lambda_{x}}\right)=V_{\delta} \subseteq X_{\delta}$, if $\delta \in S\left(\Phi_{\lambda_{x}}\right) ; P_{\delta}\left(B_{\lambda_{x}}\right)=X_{\delta}$, if $\delta \in S-S\left(\Phi_{\lambda_{x}}\right)$. Since $B_{\lambda_{x}} \subseteq U$, for any $\delta \in S-S\left(\Phi_{\lambda_{x}}\right)$, we have $P_{\delta}(U) \supseteq P_{\delta}\left(B_{\lambda_{x}}\right)=X_{\delta}$ and $P_{\delta}(U)=X_{\delta}$. On the other hand, since for any $s \in S$ and $U_{s} \in P\left(X_{s}\right),\left(\prod_{t \in S}\left(\tau_{P}\right)_{t}\right)\left(p_{s}^{-1}\left(U_{s}\right)\right) \geq$ $\left(\tau_{P}\right)_{s}\left(U_{s}\right)$, we have, for any $s \in S$,

$$
I_{P}\left(p_{s}\right)=\bigwedge_{U_{s} \in P\left(X_{s}\right)} \min \left(1,1-\left(\tau_{P}\right)_{s}\left(U_{s}\right)+\prod_{t \in S}\left(\tau_{P}\right)_{t}\left(p_{s}^{-1}\left(U_{s}\right)\right)\right)=1 .
$$

Furthermore, since by Theorem 9 in [1], we have

$$
\models \Gamma_{P}(X, \tau) \otimes I_{P}(f) \rightarrow \Gamma_{P}(f(X)),
$$

then

$$
\Gamma_{P}(U, \tau / U)=\Gamma_{P}(U, \tau / U) \otimes I_{P}\left(p_{s}\right) \leq \Gamma_{P}\left(P_{\delta}(U), \tau_{\delta}\right)=\Gamma_{P}\left(X_{\delta}, \tau_{\delta}\right) .
$$

Therefore,

$$
\bigvee_{T \sqsubset S} \bigwedge_{t \in S-T} \Gamma_{P}\left(X_{t}, \tau_{t}\right) \geq \bigwedge_{\delta \in S-S\left(\Phi_{\lambda}\right)} \Gamma_{P}\left(X_{\delta}, \tau_{\delta}\right) \geq \Gamma_{P}(U, \tau / U)>\mu .
$$

The above theorem is a generalization of the following corollary. 
Corollary 3.2. If there exists a coordinate pre-neighborhood strong compact subset $U$ of some point $x \in X$ of the product space, then all except a finite number of coordinate spaces are strong compact.

Lemma 3.3. For any fuzzifying topological space $(X, \tau), A \subseteq X$, $\vDash T_{2}^{P}(X, \tau) \rightarrow T_{2}^{P}\left(A, \tau_{/ A}\right)$.

Proof.

$$
\begin{aligned}
{\left[T_{2}^{P}(X, \tau)\right] } & =\bigwedge_{x, y \in X, x \neq y} \bigvee_{U, V \in P(X), U \cap V=\phi}\left(N_{x}^{P}(U), N_{y}^{P}(V)\right) \\
& \leq \bigwedge_{x, y \in X, x \neq y} \bigvee_{(U \cap A) \cap(V \cap A)=\phi}\left(N_{x}^{P^{A}}(U \cap A), N_{y}^{P^{A}}(V \cap A)\right) \\
& \leq \bigwedge_{x, y \in A, x \neq y} \bigvee_{U^{\prime} \cap V^{\prime}=\phi, U^{\prime}, V^{\prime} \in P(A)}\left(N_{x}^{P^{A}}\left(U^{\prime}\right), N_{y}^{P^{A}}\left(V^{\prime}\right)\right) \\
& =T_{2}^{P}(A, \tau / A),
\end{aligned}
$$

where

$$
N_{x}^{P^{A}}(U)=\bigvee_{x \in C \subseteq U} \tau_{P} / A(C) \text { and } \tau_{P} / A(B)=\bigvee_{B=V \cap A} \tau_{P}(V)
$$

Lemma 3.4. For any fuzzifying $P$-topological space $(X, \tau)$, $\models T_{2}^{P}(X, \tau) \otimes \Gamma_{P}(X, \tau) \rightarrow T_{4}^{P}(X, \tau)$.

For the definition of $T_{4}^{P}(X, \tau)$ see [3, Definition 3.1].

Proof. If $\left[T_{2}^{P}(X, \tau) \otimes \Gamma_{P}(X, \tau)\right]=0$, then the result holds. Now, suppose that $\left[T_{2}^{P}(X, \tau) \otimes \Gamma_{P}(X, \tau)\right]>\lambda>0$. Then $T_{2}^{P}(X, \tau)+\Gamma_{P}(X, \tau)-1>\lambda>0$. Therefore, from Theorem 10 in [1] $T_{2}^{P}(X, \tau) \otimes\left(\Gamma_{P}(A) \wedge \Gamma_{P}(B)\right) \wedge(A \cap B=\phi) \models^{w s} T_{2}^{P}(X, \tau) \rightarrow(\exists U)(\exists V)((U \in$ $\left.\left.\tau_{P}\right) \wedge\left(V \in \tau_{P}\right) \wedge(A \subseteq U) \wedge(B \subseteq V) \wedge(A \cap B=\phi)\right)$. Then for any $A, B \subseteq X, A \cap B=\phi$,

$$
T_{2}^{P}(X, \tau) \otimes\left(\Gamma_{P}(A) \wedge \Gamma_{P}(B)\right) \leq \bigvee_{U \cap V=\phi, A \subseteq U, B \subseteq V} \min \left(\tau_{P}(U), \tau_{P}(V)\right)
$$

or equivalently

$$
T_{2}^{P}(X, \tau) \leq \Gamma_{P}(A) \wedge \Gamma_{P}(B) \rightarrow \bigvee_{U \cap V=\phi, A \subseteq U, B \subseteq V} \min \left(\tau_{P}(U), \tau_{P}(V)\right)
$$


Hence, for any $A, B \subseteq X, A \cap B=\phi$,

$$
1-\left[\Gamma_{P}(A) \wedge \Gamma_{P}(B)\right]+\underset{U \cap V=\phi, A \subseteq U, B \subseteq V}{\bigvee} \min \left(\tau_{P}(U), \tau_{P}(V)\right)+\Gamma_{P}(X, \tau)-1>\lambda
$$

From Theorem 7 in [1] we have

$$
\models \Gamma_{P}(X, \tau) \otimes A \in \mathcal{F}_{P} \rightarrow \Gamma_{P}(A) .
$$

Then

$$
\begin{aligned}
\Gamma_{P}(X, \tau)+\left[\tau_{P}\left(A^{c}\right) \wedge \tau_{P}\left(B^{c}\right)\right]-1 & =\left(\Gamma_{P}(X, \tau)+\tau_{P}\left(A^{c}\right)-1\right) \wedge\left(\Gamma_{P}(X, \tau)+\tau_{P}\left(B^{c}\right)-1\right) \\
& \leq\left(\Gamma_{P}(X, \tau) \otimes \tau_{P}\left(A^{c}\right)\right) \wedge\left(\Gamma_{P}(X, \tau) \otimes \tau_{P}\left(B^{c}\right)\right) \\
& \leq\left[\Gamma_{P}(A) \wedge \Gamma_{P}(B)\right] .
\end{aligned}
$$

Thus,

$$
\Gamma_{P}(X, \tau)-\left[\Gamma_{P}(A) \wedge \Gamma_{P}(B)\right]-1 \leq-\left[\tau_{P}\left(A^{c}\right) \wedge \tau_{P}\left(B^{c}\right)\right] .
$$

So,

$$
1-\left[\tau_{P}\left(A^{c}\right) \wedge \tau_{P}\left(B^{c}\right)\right]+\bigvee_{U \cap V=\phi, A \subseteq U, B \subseteq V} \min \left(\tau_{P}(U), \tau_{P}(V)\right)>\lambda,
$$

i.e.,

$$
\begin{gathered}
T_{4}^{P}(X, \tau)=\bigwedge_{A \cap B=\phi} \min \left(1,1-\left[\tau_{P}\left(A^{c}\right) \wedge \tau_{P}\left(B^{c}\right)\right]\right. \\
+\bigvee_{U \cap V=\phi, A \subseteq U, B \subseteq V} \min \left(\tau_{P}(U), \tau_{P}(V)\right)>\lambda
\end{gathered}
$$

The above lemma is a generalization of the following corollary.

Corollary 3.3. Every strong compact pre-Hausdorff topological space is pre-normal.

Lemma 3.5. For any fuzzifying $P$-topological space $(X, \tau)$, $\models T_{2}^{P}(X, \tau) \otimes \Gamma_{P}(X, \tau) \rightarrow T_{3}^{P}(X, \tau)$. For the definition of $T_{3}^{P}(X, \tau)$ see $[3$, Definition 3.1]. 
Proof. Immediate, set $A=\{x\}$ in the above lemma.

The above lemma is a generalization of the following corollary.

Corollary 3.4. Every strong compact pre-Hausdorff topological space is pre-regular.

Theorem 3.3. For any fuzzifying topological space $(X, \tau)$ and $A \subseteq X$, $\models T_{2}^{P}(X, \tau) \otimes \Gamma_{P}(A) \rightarrow A \in \mathcal{F}_{P}$.

Proof. For any $\{x\} \subset A^{c}$, we have $\{x\} \cap A=\phi$ and $\Gamma_{P}(\{x\})=1$. By Theorem 10 in [1]

$$
\left.\left[T_{2}^{P}(X, \tau) \otimes\left(\Gamma_{P}(A) \wedge \Gamma_{P}(\{x\})\right)\right] \leq \bigvee_{G \cap H_{x}=\phi, A \subseteq G, x \in H_{x}} \min \left(\tau_{P}(G), \tau_{P}\left(H_{x}\right)\right)\right)
$$

Assume

$$
\beta_{x}=\left\{H_{x}: A \cap H_{x}=\phi, x \in H_{x}\right\}, \bigcup_{x \in X \backslash A} f(x) \supseteq A^{c}
$$

and

So, $\bigcup_{x \in A^{c}} f(x)=A^{c}$.

$$
\bigcup_{x \in A^{c}} f(x) \cap A=\bigcup_{x \in A^{c}}(f(x) \cap A)=\phi .
$$

Therefore,

$$
\begin{aligned}
& {\left[T_{2}^{P}(X, \tau) \otimes \Gamma_{P}(A)\right] \leq \bigvee_{G \cap H_{x}=\phi, A \subseteq G, x \in H_{x}} \tau_{P}\left(H_{x}\right)} \\
& \leq \bigwedge_{x \in A^{c}} \bigvee_{A \cap H_{x}=\phi, x \in H_{x}} \tau_{P}\left(H_{x}\right)
\end{aligned}
$$

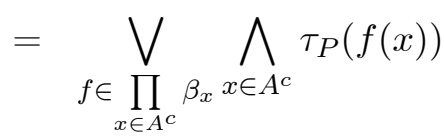

$$
\begin{aligned}
& \leq \bigvee_{f \in \prod_{x \in A^{c}} \beta_{x}} \tau_{P}\left(\bigcup_{x \in A^{c}} f(x)\right) \\
& =\bigvee_{f \in \prod_{x \in X \backslash A} \beta_{x}} \tau_{P}\left(A^{c}\right)=\mathcal{F}_{P}(A) .
\end{aligned}
$$

The above theorem is a generalization of the following corollary.

Corollary 3.5. Strong compact subspace of a pre-Hausdorff topological space is pre-closed. 


\section{Fuzzifying locally strong compactness}

Definition 4.1. Let $\Omega$ be a class of fuzzifying topological spaces. A unary fuzzy predicate $L_{P} C: \Omega \rightarrow[0,1]$, called fuzzifying locally strong compactness, is given as follows:

$$
(X, \tau) \in L_{P} C:=(\forall x)(\exists B)\left(\left(x \in \operatorname{Int}_{P}(B) \otimes \Gamma_{P}(B, \tau / B)\right) .\right.
$$

Since $\left[x \in \operatorname{Int}_{P}(X)\right]=N_{x}^{P}(X)=1$, then $L_{P} C(X, \tau) \geq \Gamma_{P}(X, \tau)$. Therefore, $\mid=(X, \tau) \in \Gamma_{P} \rightarrow(X, \tau) \in L_{P} C$.

Also, since $\models(X, \tau) \in \Gamma \rightarrow(X, \tau) \in L C$ [21] and $\models(X, \tau) \in \Gamma_{P} \rightarrow$ $(X, \tau) \in \Gamma[1], \models(X, \tau) \in \Gamma_{P} \rightarrow(X, \tau) \in L C$.

Theorem 4.1. For any fuzzifying topological space $(X, \tau)$ and $A \subseteq X$, $\models(X, \tau) \in L_{P} C \otimes A \in F_{P} \rightarrow(A, \tau / A) \in L_{P} C$.

Proof. We have

$$
L_{P} C(X, \tau)=\bigwedge_{x \in X} \bigvee_{B \subseteq X} \max \left(0, N_{x}^{P^{X}}(B)+\Gamma_{P}(B, \tau / B)-1\right)
$$

and

$$
L_{P} C(A, \tau / A)=\bigwedge_{x \in A} \bigvee_{G \subseteq A} \max \left(0, N_{x}^{P^{A}}(G)+\Gamma_{P}(G,(\tau / A) / G)-1\right) .
$$

Now, suppose that $\left[(X, \tau) \in L_{P} C \otimes A \in F_{P}\right]>\lambda>0$. Then for any $x \in A$, there exists $B \subseteq X$ such that

$$
N_{x}^{P^{X}}(B)+\Gamma_{P}(B, \tau / B)+\tau_{P}(X-A)-2>\lambda
$$

Set $E=A \cap B \in P(A)$. Then,

$$
N_{x}^{P^{A}}(E)=\bigvee_{E=C \cap B} N_{x}^{P^{X}}(C) \geq N_{x}^{P^{X}}(B)
$$

and for any $U \in P(E)$, we have

$$
\begin{aligned}
\left(\tau_{P} / A\right)_{P} / E(U) & =\bigvee_{U=C \cap E} \tau_{P} / A(C) \\
& =\bigvee_{U=C \cap E C=D \cap A} \bigvee_{P} \tau_{P}(D) \\
& =\bigvee_{U=D \cap A \cap E} \tau_{P}(D)=\bigvee_{U=D \cap E} \tau_{P}(D) .
\end{aligned}
$$


Similarly,

$$
\left(\tau_{P} / B\right)_{P} / E(U)=\bigvee_{U=D \cap E} \tau_{P}(D) .
$$

Thus, $\left(\tau_{P} / B\right)_{P} / E=\left(\tau_{P} / A\right)_{P} / E$ and $\Gamma_{P}(E,(\tau / A) / E)=\Gamma_{P}(E,(\tau / B) / E)$. Furthermore,

$$
\begin{aligned}
{\left[E \in F_{P} / B\right] } & =\tau_{P} / B(B-E)=\tau_{P} / B\left(B \cap E^{c}\right) \\
& =\bigvee_{B \cap E^{c}=B \cap D} \tau_{P}(D) \\
& \geq \tau_{P}(X-A)=F_{P}(A) .
\end{aligned}
$$

Since $\models(X, \tau) \in \Gamma_{P} \otimes A \in F_{P} \rightarrow(A, \tau / A) \in \Gamma_{P}$ (see [1], Theorem 7], from (4.1) we have for any $x \in A$ that

$$
\begin{aligned}
& \bigvee_{G \subseteq A} \max \left(0, N_{x}^{P^{A}}(G)+\Gamma_{P}(G,(\tau / A) / G)-1\right) \\
\geq & N_{x}^{P^{A}}(E)+\Gamma_{P}(E,(\tau / A) / E)-1 \\
= & N_{x}^{P^{A}}(E)+\Gamma_{P}(E,(\tau / B) / E)-1 \\
\geq & N_{x}^{P^{X}}(B)+\left[\Gamma_{P}(B, \tau / B) \otimes E \in F_{P} / B\right]-1 \\
\geq & N_{x}^{P^{X}}(B)+\Gamma_{P}(B, \tau / B)+\left[E \in F_{P} / B\right]-2 \\
\geq & N_{x}^{P^{X}}(B)+\Gamma_{P}(B, \tau / B)+\left[A \in F_{P}\right]-2>\lambda .
\end{aligned}
$$

Therefore,

$$
L_{P} C(A, \tau / A)=\bigwedge_{x \in A} \bigvee_{G \subseteq A} \max \left(0, N_{x}^{P^{A}}(G)+\Gamma_{P}(G,(\tau / A) / G)-1\right)>\lambda .
$$

Hence, $\left[(X, \tau) \in L_{P} C \otimes A \in F_{P}\right] \leq L_{P} C(A, \tau / A)$.

As a crisp result of the above theorem we have the following corollary.

Corollary 4.1. Let $A$ be a pre-closed subset of locally strong compact space $(X, \tau)$. Then $A$ with the relative topology $\tau / A$ is locally strong compact.

The following theorem is a generalization of the statement "If $X$ is a preHausdorff topological space and $A$ is a pre-dense locally strong compact subspace, then $A$ is pre-open", where $A$ is a pre-dense in a topological space $X$ if and only if the pre-closure of $A$ is $X$.

Theorem 4.2. For any fuzzifying $P$-topological space $(X, \tau)$ and $A \subseteq X$,

$$
\models T_{2}^{P}(X, \tau) \otimes L_{P} C(A, \tau / A) \otimes\left(C l_{P}(A) \equiv X\right) \rightarrow A \in \tau_{P}
$$


Proof. Assume

$$
\left[T_{2}^{P}(X, \tau) \otimes L_{P} C(A, \tau / A) \otimes\left(C l_{P}(A) \equiv X\right)\right]>\lambda>0 .
$$

Then,

$$
L_{P} C(A, \tau / A)>\lambda-\left[T_{2}^{P}(X, \tau) \otimes\left(C l_{P}(A) \equiv X\right)\right]+1=\lambda^{\prime}>\lambda,
$$

i. e.,

$$
\bigwedge_{x \in A} \bigvee_{B \subseteq A} \max \left(0, N_{x}^{P^{A}}(B)+\Gamma_{P}(B,(\tau / A) / B)-1\right)>\lambda^{\prime}
$$

Thus, for any $x \in A$, there exists $B_{x} \subseteq A$ such that

$$
N_{x}^{P^{A}}\left(B_{x}\right)+\Gamma_{P}\left(B_{x},(\tau / A) / B_{x}\right)-1>\lambda^{\prime} .
$$

i.e.,

$$
\bigvee_{H \cap A=B_{x}} \bigvee_{x \in K \subseteq H} \tau_{P}(K)+\Gamma_{P}\left(B_{x},(\tau / A) / B_{x}\right)-1>\lambda^{\prime} .
$$

Hence, there exists $K_{x}$ such that

$$
K_{x} \cap A=B_{x}, \tau_{P}\left(K_{x}\right)+\Gamma_{P}\left(B_{x},(\tau / A) / B_{x}\right)-1>\lambda^{\prime} .
$$

Therefore, $\tau_{P}\left(K_{x}\right)>\lambda^{\prime}$.

(1) If for any $x \in A$ there exists $K_{x}$ such that

$$
x \in K_{x} \subseteq B_{x} \subseteq A \text {, then } \bigcup_{x \in A} K_{x}=A
$$

and

$$
\tau_{P}(A)=\tau_{P}\left(\bigcup_{x \in A} K_{x}\right) \geq \bigwedge_{x \in A} \tau_{P}\left(K_{x}\right) \geq \lambda^{\prime}>\lambda .
$$

(2) If there exists $x_{\circ} \in A$ such that

$$
K_{x_{\circ}} \cap\left(B_{x_{\circ}}^{c}\right) \neq \phi, \tau_{P}\left(K_{x_{\circ}}\right)+\Gamma_{P}\left(B_{x_{\circ}},(\tau / A) / B_{x_{\circ}}\right)-1>\lambda^{\prime} .
$$

From the hypothesis

$$
\left[T_{2}^{P}(X, \tau) \otimes L_{P} C(A, \tau / A) \otimes\left(C l_{P}(A) \equiv X\right)\right]>\lambda>0,
$$

we have $\left[T_{2}^{P}(X, \tau) \otimes\left(C l_{P}(A) \equiv X\right)\right] \neq 0$. So

$\tau_{P}\left(K_{x_{\circ}}\right)+\Gamma_{P}\left(B_{x_{\circ}},(\tau / A) / B_{x_{\circ}}\right)-1+\left[T_{2}^{P}(X, \tau) \otimes\left(C l_{P}(A) \equiv X\right)\right]-1>0$. 
Therefore,

$\tau_{P}\left(K_{x_{\circ}}\right)+\Gamma_{P}\left(B_{x_{\circ}},(\tau / A) / B_{x_{\circ}}\right)-1+T_{2}^{P}(X, \tau)+\left[\left(C l_{P}(A) \equiv X\right)\right]-1-1>\lambda$.

Since

$$
\begin{aligned}
&\left(\tau_{P} / A\right)_{P} / B_{x_{\circ}}(U)=\bigvee_{U=C \cap B_{x_{\diamond}}} \tau_{P} / A(C) \\
&=\bigvee_{U=C \cap B_{x_{\diamond}}} \bigvee_{C=D \cap A} \tau_{P}(D) \\
&=\bigvee_{U=D \cap B_{x_{\circ}}} \tau_{P}(D)=\tau_{P} / B_{x_{\circ}}(U), \\
& \Gamma_{P}\left(B_{x_{\circ}},(\tau / A) / B_{x_{\circ}}\right)=\Gamma_{P}\left(B_{x_{\circ}}, \tau / B_{x_{\circ}}\right) .
\end{aligned}
$$

From Theorem 3.3 we have

$$
\begin{aligned}
\tau_{P}\left(B_{x_{\diamond}}^{c}\right) & \geq T_{2}^{P}(X, \tau) \otimes \Gamma_{P}\left(B_{x_{\circ}}, \tau / B_{x_{\circ}}\right) \\
& \geq T_{2}^{P}(X, \tau)+\Gamma_{P}\left(B_{x_{\diamond}}, \tau / B_{x_{\diamond}}\right)-1 .
\end{aligned}
$$

Hence,

$$
\tau_{P}\left(K_{x_{\circ}}\right)+\tau_{P}\left(B_{x_{\circ}}^{c}\right)+\left[C l_{P}(A) \equiv X\right]-2>\lambda .
$$

Now, for any $y \in A^{c}$ we have

$$
\left[C l_{P}(A) \equiv X\right]=\bigwedge_{x \in X}\left(1-N_{x}^{P^{X}}\left(A^{c}\right)\right) \leq 1-N_{y}^{P^{X}}\left(A^{c}\right) .
$$

Since $(X, \tau)$ is a fuzzifying $P$-topological space,

$$
\begin{aligned}
\tau_{P}\left(K_{x_{\circ}}\right)+\tau_{P}\left(B_{x_{\circ}}^{c}\right)-1 & \leq \tau_{P}\left(K_{x_{\circ}}\right) \otimes \tau_{P}\left(B_{x_{\circ}}^{c}\right) \\
& \leq \tau_{P}\left(K_{x_{\diamond}}\right) \wedge \tau_{P}\left(B_{x_{\diamond}}^{c}\right) \\
& \leq \tau_{P}\left(K_{x_{\diamond}} \cap B_{x_{\circ}}^{c}\right) \\
& \leq N_{y}^{P^{X}}\left(K_{x_{\diamond}} \cap B_{x_{\circ}}^{c}\right) \leq N_{y}^{P^{X}}\left(A^{c}\right),
\end{aligned}
$$

where

$$
\begin{aligned}
y \in K_{x_{\diamond}} \cap B_{x_{\diamond}}^{c} \subseteq H_{x_{\diamond}} \cap\left(H_{x_{\diamond}} \cap A\right)^{c} & =H_{x_{\diamond}} \cap\left(H_{x_{\circ}}^{c} \cup A^{c}\right) \\
& =H_{x_{\diamond}} \cap A^{c} \subseteq A^{c} .
\end{aligned}
$$

Therefore,

$$
\begin{aligned}
0 & <\lambda<\tau_{P}\left(K_{x_{\circ}}\right)+\tau_{P}\left(B_{x_{\circ}}^{c}\right)+\left[C l_{P}(A) \equiv X\right]-2 \\
& =\tau_{P}\left(K_{x_{\circ}}\right)+\tau_{P}\left(B_{x_{\diamond}}^{c}\right)-1+\left[C l_{P}(A) \equiv X\right]-1 \\
& \leq N_{y}^{P^{X}}\left(A^{c}\right)+1-N_{y}^{P^{X}}\left(A^{c}\right)-1=0,
\end{aligned}
$$

a contradiction. So, case (2) does not hold. We complete the proof. 
Theorem 4.3. For any fuzzifying $P$-topological space $(X, \tau)$,

$$
\begin{gathered}
\models T_{2}^{P}(X, \tau) \otimes\left(L_{P} C(X, \tau)\right)^{2} \\
\rightarrow \forall x \forall U\left(U \in N_{x}^{P^{X}} \rightarrow \exists V\left(V \in N_{x}^{P^{X}} \wedge C l_{P}(V) \subseteq U \wedge \Gamma_{P}(V)\right)\right),
\end{gathered}
$$

where $\left(L_{P} C(X, \tau)\right)^{2}:=L_{P} C(X, \tau) \otimes L_{P} C(X, \tau)$.

Proof. We need to show that for any $x$ and $U, x \in U$,

$$
\begin{gathered}
T_{2}^{P}(X, \tau) \otimes\left(L_{P} C(X, \tau)\right)^{2} \otimes N_{x}^{P^{X}}(U) \\
\leq \bigvee_{V \subseteq X}\left(N_{x}^{P^{X}}(V) \wedge \bigwedge_{y \in U^{c}} N_{x}^{P^{X}}\left(V^{c}\right) \wedge \Gamma_{P}(V, \tau / V)\right)
\end{gathered}
$$

Assume that $T_{2}^{P}(X, \tau) \otimes\left(L_{P} C(X, \tau)\right)^{2} \otimes N_{x}^{P^{X}}(U)>\lambda>0$. Then, for any $x \in X$ there exists $C$ such that

(4.2) $T_{2}^{P}(X, \tau)+N_{x}^{P^{X}}(C)+\left(L_{P} C(X, \tau)\right)^{2}+N_{x}^{P^{X}}(U)-3>\lambda$.

Since $(X, \tau)$ is fuzzifying $P$-topological space,

$$
\begin{aligned}
& N_{x}^{P^{X}}(C)+N_{x}^{P^{X}}(U)-1 \leq N_{x}^{P^{X}}(C) \otimes N_{x}^{P^{X}}(U) \\
\leq & N_{x}^{P^{X}}(C) \wedge N_{x}^{P^{X}}(U) \\
\leq & N_{x}^{P^{X}}(C \cap U)=\bigvee_{x \in W \subseteq C \cap U} \tau_{P}(W) .
\end{aligned}
$$

Therefore, there exists $W$ such that $x \in W \subseteq C \cap U$, and $T_{2}^{P}(X, \tau)+$ $\left(L_{P} C(X, \tau)\right)^{2}+\tau_{P}(W)-2>\lambda$. By Lemmas 3.3 and 3.5 we have $T_{2}^{P}(X, \tau) \leq$ $T_{2}^{P}(C, \tau / C)$ and

$T_{2}^{P}(C, \tau / C)+\Gamma_{P}(C, \tau / C)-1 \leq T_{2}^{P}(C, \tau / C) \otimes \Gamma_{P}(C, \tau / C) \leq T_{3}^{P}(C, \tau / C)$.

Thus, $T_{3}^{P}(X, \tau)+\Gamma_{P}(C, \tau / C)+\tau_{P}(W)-2>\lambda$. Since for any $x \in W \subseteq U$, we have

$$
T_{3}^{P}(C, \tau / C) \leq 1-\tau_{P} / C(W)+\bigvee_{G \subseteq C}\left(\left(N_{x}^{P^{C}}(G) \wedge \bigwedge_{y \in C-W} N_{y}^{P^{C}}(C-G)\right)\right)
$$


so there exists $G, x \in G \subseteq W$ such that

$$
\begin{array}{r}
\left(\left(N_{x}^{P^{C}}(G) \wedge \bigwedge_{y \in C-W} N_{y}^{P^{C}}(C-G)\right)\right) \\
\geq T_{3}^{P}(C, \tau / C)+\tau_{P} / C(W)-1 \geq T_{3}^{P}(C, \tau / C)+\tau_{P}(W)-1
\end{array}
$$

and

$$
\left(\left(N_{x}^{P^{C}}(G) \wedge \bigwedge_{y \in C-W} N_{y}^{P^{C}}(C-G)\right)\right)+\Gamma_{P}(C, \tau / C)-1>\lambda .
$$

Thus,

$$
N_{x}^{P^{C}}(G)=\bigvee_{D \cap C=G} N_{x}^{P^{X}}(D)=N_{x}^{P^{X}}\left(G \cup C^{c}\right)>\lambda^{\prime}=\lambda+1-\Gamma_{P}(C, \tau / C) \geq \lambda .
$$

Furthermore, for any $y \in C-W$,

$$
N_{y}^{P^{C}}(C-G)=\bigvee_{D \cap C=C \cap G^{c}} N_{y}^{P^{X}}\left(G^{c} \cup C^{c}\right)=N_{y}^{P^{X}}\left(G^{c}\right)>\lambda^{\prime}
$$

and

$$
N_{x}^{P^{X}}(G)=N_{x}^{P^{X}}\left(\left(G \cup C^{c}\right) \cap C\right) \geq N_{x}^{P^{X}}\left(G^{c}\right) \wedge N_{x}^{P^{X}}(C)>\lambda^{\prime} .
$$

Since $N_{y}^{P^{X}}\left(G^{c}\right)=\bigvee_{x \in B^{c} \subseteq G^{c}} \tau_{P}\left(B^{c}\right)>\lambda^{\prime}$, for any $y \in C-W$, there exists $B_{y}^{c}$ such that $y \in B_{y}^{c} \subseteq G^{c}$ and $\tau_{P}\left(B_{y}^{c}\right)>\lambda^{\prime}$. Set $B^{c}=\bigcup_{y \in C-W} B_{y}^{c}$. Then $C-W \subseteq B^{c} \subseteq G^{c}$ and

$$
\tau_{P}\left(B^{c}\right) \geq \bigwedge_{y \in C-W} \tau_{P}\left(B_{y}^{c}\right) \geq \lambda^{\prime} .
$$

Again, set $V=B \cap C$, then $V \subseteq(C-W)^{c} \cap C=\left(C^{c} \cup W\right) \cap C=C \cap W=$ $W \subseteq U \cap C$ and $V^{c}=B^{c} \cup C^{c}$. Since $(X, \tau)$ is fuzzifying $P$-topological space,

$$
\begin{aligned}
N_{x}^{P^{X}}(V)=N_{x}^{P^{X}}(B \cap C) & \geq N_{x}^{P^{X}}(B) \wedge N_{x}^{P^{X}}(C) \\
& \geq N_{x}^{P^{X}}(G) \wedge N_{x}^{P^{X}}(C)>\lambda .
\end{aligned}
$$

By (4.2) and Theorem 3.3,

$$
\tau_{P}\left(C^{c}\right) \geq T_{2}^{P}(X, \tau) \otimes \Gamma_{P}(C, \tau / C) \geq T_{2}^{P}(X, \tau)+\Gamma_{P}(C, \tau / C)-1 \geq \lambda^{\prime} .
$$


So

i.e., $\tau_{P}\left(V^{c}\right)+\Gamma_{P}(C, \tau / C)-1 \geq \lambda$ and

$\Gamma_{P}(V, \tau / V)=\Gamma_{P}(V,(\tau / C) / V)$

$$
\begin{aligned}
& \geq \tau_{P} / C(C-V)+\Gamma_{P}(C, \tau / C)-1 \\
& \geq \tau_{P}\left(V^{c}\right)+\Gamma_{P}(C, \tau / C)-1 \geq \lambda
\end{aligned}
$$

Finally,

$$
\bigwedge_{y \in U^{c}} N_{y}^{P^{X}}\left(V^{c}\right) \geq \bigwedge_{y \in V^{c}} N_{y}^{P^{X}}\left(V^{c}\right)=\tau_{P}\left(V^{c}\right) \geq \lambda
$$

Thus, by (4.3), (4.4) and (4.5), for any $x \in U$, there exists $V \subseteq U$ such that $N_{x}^{P^{X}}(V)>\lambda, \bigwedge_{y \in U^{c}} N_{y}^{P^{X}}\left(V^{c}\right) \geq \lambda$ and $\Gamma_{P}(V, \tau / V) \geq \lambda$. So $\underset{V \subseteq X}{\bigvee}\left(N_{x}^{P^{X}}(V) \wedge\right.$ $\left.\bigwedge_{y \in U^{c}} N_{y}^{P^{X}}\left(V^{c}\right) \wedge \Gamma_{P}(V, \tau / V)\right) \geq \lambda$.

Theorem 4.4. For any fuzzifying $P$-topological space $(X, \tau)$,

$$
\models T_{2}^{P}(X, \tau) \otimes\left(L_{P} C(X, \tau)\right)^{2} \rightarrow T_{3}^{P}(X, \tau) .
$$

Proof. By Theorem 4.3, for any $x \in U$, we have

$$
\begin{gathered}
\bigvee_{x \in V \subseteq U}\left(N_{x}^{P^{X}}(V) \wedge \bigwedge_{y \in U^{c}} N_{y}^{P^{X}}\left(V^{c}\right)\right. \\
\geq\left[T_{2}^{P}(X, \tau) \otimes\left(\Gamma_{P}(C, \tau / C)\right)^{2} \otimes N_{x}^{P^{X}}(U)\right] .
\end{gathered}
$$

Thus,

$$
\begin{gathered}
1-N_{x}^{P^{X}}(U)+\bigvee_{x \in V \subseteq U}\left(N_{x}^{P^{X}}(V) \wedge \bigwedge_{y \in U^{c}} N_{y}^{P^{X}}\left(V^{c}\right)\right. \\
\geq\left[T_{2}^{P}(X, \tau) \otimes\left(\Gamma_{P}(C, \tau / C)\right)^{2}\right]
\end{gathered}
$$

i.e.,

$$
\left[T_{3}^{P}(X, \tau)\right] \geq\left[T_{2}^{P}(X, \tau) \otimes\left(\Gamma_{P}(C, \tau / C)\right)^{2}\right]
$$

Theorem 4.5. For any fuzzifying $P$-topological space $(X, \tau)$,

$$
\begin{aligned}
\models T_{3}^{P}(X, \tau) \otimes L_{P} C(X, \tau) & \rightarrow \forall A \forall U\left(U \in N_{A}^{P^{X}} \otimes \Gamma_{P}(A, \tau / A)\right. \\
& \left.\rightarrow \exists V\left(V \subseteq U \wedge U \in N_{A}^{P^{X}} \wedge \tau_{P}\left(V^{c}\right) \wedge \Gamma_{P}(V, \tau / V)\right)\right),
\end{aligned}
$$

where

$$
U \in N_{A}^{P^{X}}:=(\forall x)\left(x \in A \wedge U \in N_{x}^{P^{X}}\right) .
$$


Proof. We only need to show that for any $A, U \in P(X)$,

$$
\begin{gathered}
{\left[T_{3}^{P}(X, \tau) \otimes L_{P} C(X, \tau) \otimes \Gamma_{P}(A, \tau / A) \otimes N_{A}^{P^{X}}(U)\right]} \\
\quad \leq \bigvee_{V \subseteq U}\left(N_{A}^{P^{X}}(V) \wedge \tau_{P}\left(V^{c}\right) \wedge \Gamma_{P}(V, \tau / V)\right) .
\end{gathered}
$$

Indeed, if

$$
\left[T_{3}^{P}(X, \tau) \otimes L_{P} C(X, \tau) \otimes \Gamma_{P}(A, \tau / A) \otimes N_{A}^{P^{X}}(U)\right]>\lambda>0,
$$

then for any $x \in A$, there exists $C \in P(X)$ such that

$$
\left[T_{3}^{P}(X, \tau) \otimes N_{x}^{P^{X}}(C) \otimes \Gamma_{P}(C, \tau / C) \otimes \Gamma_{P}(A, \tau / A) \otimes N_{A}^{P^{X}}(U)\right]>\lambda .
$$

Since $(X, \tau)$ is fuzzifying $P$-topological space,

$$
\begin{aligned}
\bigvee_{x \in W \subseteq C \cap U} \tau_{P}(W)=N_{x}^{P^{X}}(C \cap U) \geq & N_{x}^{P^{X}}(C) \wedge N_{x}^{P^{X}}(U) \\
& \geq N_{x}^{P^{X}}(C) \wedge N_{A}^{P^{X}}(U) \\
& \geq N_{x}^{P^{X}}(C) \otimes N_{A}^{P^{X}}(U) .
\end{aligned}
$$

Then, there exists $W$ such that $x \in W \subseteq C \cap U$, and

$$
\left[T_{3}^{P}(X, \tau) \otimes \tau_{P}(W) \otimes \Gamma_{P}(C, \tau / C) \otimes \Gamma_{P}(A, \tau / A)\right]>\lambda .
$$

Therefore,

$\left.(4.6)\left[T_{3}^{P}(X, \tau)\right]+\tau_{P}(W)-1>\lambda+2-\Gamma_{P}(C, \tau / C)-\Gamma_{P}(A, \tau / A)\right]=\lambda^{\prime} \geq \lambda$.

Since for any $x \in W$,

$$
\left[T_{3}^{P}(X, \tau)\right] \leq 1-\tau_{P}(W)+\bigvee_{B \subseteq W}\left(N_{x}^{P^{X}}(B) \wedge \bigwedge_{y \in W^{c}} N_{y}^{P^{X}}\left(B^{c}\right)\right)
$$

we have

$$
\bigvee_{B \subseteq W}\left(N_{x}^{P^{X}}(B) \wedge \bigwedge_{y \in W^{c}} N_{y}^{P^{X}}\left(B^{c}\right)\right)>\lambda^{\prime}
$$

Thus, there exists $B_{x}$ such that $x \in B_{x} \subseteq W \subseteq C \cap U$ and for any $y \in W^{c}$, we have

$$
N_{y}^{P^{X}}\left(B_{x}^{c}\right)>\lambda^{\prime}, \quad N_{x}^{P^{X}}\left(B_{x}\right)>\lambda^{\prime} .
$$


Since

$$
N_{y}^{P^{X}}\left(B_{x}^{c}\right)=\bigvee_{x \in G^{c} \subseteq B_{x}^{c}} \tau_{P}\left(G^{c}\right)>\lambda^{\prime},
$$

then for any $y \in W^{c}$, there exists $G_{x y}$ such that

$$
x \in G_{x y}^{c} \subseteq B_{x}^{c} \text { and } \tau_{P}\left(G_{x y}^{c}\right)>\lambda^{\prime} .
$$

Set

$$
G_{x}^{c}=\bigcup_{y \in W^{c}} G_{x y}^{c}
$$

then $W^{c} \subseteq G_{x y}^{c} \subseteq B_{x}^{c}$ and $\tau_{P}\left(G_{x}^{c}\right) \geq \bigwedge_{y \in W^{c}} \tau_{P}\left(G_{x y}^{c}\right) \geq \lambda^{\prime}$. Since $G_{x} \supseteq B_{x}$, $N_{x}^{P^{X}}\left(G_{x}\right) \geq N_{x}^{P^{X}}\left(B_{x}\right)>\lambda^{\prime}$, i.e., $\underset{x \in H \subseteq G_{x}}{\bigvee} \tau_{P}(H)>\lambda^{\prime}$. Thus, there exists $H_{x}$ such that $x \in H_{x} \subseteq G_{x}$ and $\tau_{P}\left(H_{x}\right)>\lambda^{\prime}$. Hence, for any $x \in A$, there exists $H_{x}$ and $G_{x}$ such that $x \in H_{x} \subseteq G_{x} \subseteq U, \tau_{P}\left(H_{x}\right)>\lambda^{\prime}$ and $W \supseteq \bigcup_{x \in A} G_{x} \supseteq \bigcup_{x \in A} H_{x} \supseteq A$. We define $\Re \in \Im(P(A))$ as follows:

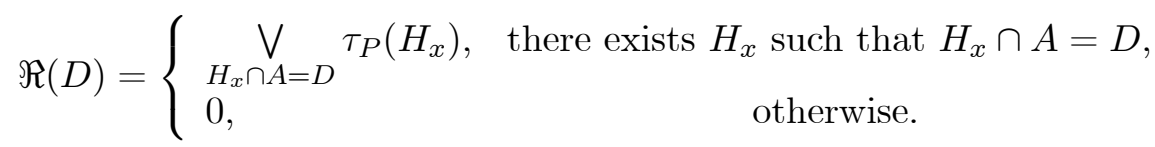

Let $\Gamma_{P}(A, \tau / A)=\mu>\mu-\epsilon(\epsilon>0)$. Then $1-K_{P}(\Re, A)+\bigvee_{\wp \leq \Re}[K(\Re, A) \otimes$ $F F(\wp)]>\mu-\epsilon$, where

$$
\begin{gathered}
{[K(\Re, A)]=\bigwedge_{x \in A} \bigvee_{x \in B} \Re(B)=\bigwedge_{x \in A} \bigvee_{x \in D} \Re(D)} \\
=\bigwedge_{x \in A} \bigvee_{x \in D} \bigvee_{H_{x^{\prime}} \cap A=D} \tau_{P}\left(H_{x^{\prime}}\right) \geq \lambda^{\prime}
\end{gathered}
$$

and $\left[\Re \subseteq \tau_{P} \backslash A\right]=\bigwedge_{B \subseteq X} \min \left(1,1-\Re(B)+\tau_{P} \backslash A(B)\right)=\bigwedge_{B \subseteq X} \min (1,1-$ $\left.\underset{H_{x} \cap A=B}{\bigvee} \tau_{P}\left(H_{x}\right)+\underset{H \cap A=B}{\bigvee} \tau_{P}(H)\right)=1$. So, $K_{P}(\Re, A)=[K(\Re, A)] \geq \lambda^{\prime}$. By (4.6), $[K(\Re, A) \otimes F F(\wp)]>\mu-\epsilon-1+K_{P}(\Re, A) \geq \mu-\epsilon-1+\lambda^{\prime} \geq \lambda-\epsilon$.

Thus, $\bigwedge_{x \in A} \bigvee_{x \in E} \Re(E)+1-\bigwedge\left\{\delta: F\left(\wp_{\delta}\right)\right\}-1>\lambda-\epsilon$, and $\bigwedge_{x \in A} \bigvee_{x \in E} \Re(E)>\lambda-\epsilon+\bigwedge\left\{\delta: F\left(\wp_{\delta}\right)\right\}$. 
Hence, there exists $\beta>0$ such that $F\left(\wp_{\beta}\right)$ and $\bigwedge_{x \in A} \bigvee_{x \in D} \Re(D)>\lambda-\epsilon+\beta$. Therefore for any $x \in A$, there exists $D_{x} \subseteq A$ such that $\wp\left(D_{x}\right)>\lambda-\epsilon+\beta$ and $\bigcup_{x \in A} D_{x} \supseteq A$. Suitably choose $\epsilon$ such that $\lambda-\epsilon>0$, then $\wp\left(D_{x}\right)>$ $\beta>0$. Since $\Re\left(D_{x}\right) \geq \wp\left(D_{x}\right)>0, D_{x}=H_{x^{\prime}} \cap A$, i.e., $H_{x^{\prime}} \cap A \in \wp \beta$. By $F(\wp \beta)$, there exists finite $H_{x_{1}^{\prime}}, H_{x_{2}^{\prime}}, \ldots, H_{x_{n}^{\prime}}$ such that $\bigcup_{i=1}^{n} H_{x_{i}^{\prime}} \supseteq A$ and $\bigcup_{i=1}^{n} H_{x_{i}^{\prime}} \subseteq \bigcup_{i=1}^{n} G_{x_{i}^{\prime}}$.

Set $V=\bigcup_{i=1}^{n} G_{x_{i}^{\prime}}$, and $V^{c}=\bigcap_{i=1}^{n} G_{x_{i}^{\prime}}^{c}, A \subseteq V \subseteq U$, and $\tau_{P}\left(V^{c}\right) \geq$ $\bigwedge_{1 \leq i \leq n} \tau_{P}\left(G_{x_{i}^{\prime}}^{c}\right) \geq \lambda^{\prime}>\lambda$. Since for any $x \in A, G_{x} \subseteq W \subseteq C \cap U \subseteq C$, we have $V=\bigcup_{i=1}^{n} G_{x_{i}^{\prime}} \subseteq W \subseteq C$. Because $\tau_{P} \backslash C(C-V)=\underset{D \cap C=C \cap V^{c}}{\bigvee} \tau_{P}(D) \geq$ $\tau_{P}\left(V^{c}\right) \geq \lambda^{\prime}$. Thus by (4.6), $\tau_{P} \backslash C(C-V)+\Gamma_{P}(C, \tau / C)-1>\lambda$. By Theorem 5.1 in $[24], \Gamma_{P}(V, \tau / V)=\Gamma_{P}(V, \tau / C / V) \geq\left[\Gamma_{P}(C, \tau / C) \otimes \tau_{P} \backslash C(C-V)\right]>$ $\lambda$.

Finally, we have for any $x \in A$,

$N_{x}^{P^{X}}(V)=N_{x}^{P^{X}}\left(\bigcup_{i=1}^{n} G_{x_{i}^{\prime}}\right) \geq N_{x}^{P^{X}}\left(\bigcup_{i=1}^{n} H_{x_{i}^{\prime}}\right) \geq \tau_{P}\left(\bigcup_{i=1}^{n} H_{x_{i}^{\prime}}\right) \geq \bigwedge_{1 \leq i \leq n} \tau_{P}\left(H_{x_{i}^{\prime}}\right) \geq$ $\lambda^{\prime}>\lambda$. So, $N_{A}^{P^{X}}(V)=\bigwedge_{x \in A} N_{x}^{P^{X}}(V) \geq \lambda$. Therefore, $N_{A}^{P^{X}}(V) \wedge \tau_{P}\left(V^{c}\right) \wedge$ $\Gamma_{P}(V, \tau / V) \geq \lambda$.

Thus, $\underset{V \subseteq U}{\bigvee}\left(N_{A}^{P^{X}}(V) \wedge \tau_{P}\left(V^{c}\right) \wedge \Gamma_{P}(V, \tau / V)\right) \geq \lambda$.

Theorem 4.6. Let $(X, \tau)$ and $(Y, \sigma)$ be two fuzzifying topological space and $f \in Y^{X}$ be surjective. Then $=L_{P} C(X, \tau) \otimes C_{P}(f) \otimes O(f) \rightarrow L C(Y, \sigma)$. For the definition of $O(f)$, see [32].

Proof. If $\left[L_{P} C(X, \tau) \otimes C_{P}(f) \otimes O(f)\right]>\lambda>0$, then for any $x \in X$, there exists $U \subseteq X$, such that $\left[N_{x}^{P^{X}}(U) \otimes \Gamma_{P}(U, \tau / U) \otimes C_{P}(f) \otimes O(f)\right]>\lambda$. Since $N_{x}^{P^{X}}(U)=\bigvee_{x \in V \subseteq U} \tau_{P}(V)$, so there exists $V^{\prime} \subseteq X$ such that $x \in V^{\prime} \subseteq U$ and $\left[\tau_{P}\left(V^{\prime}\right) \otimes \Gamma_{P}(U, \tau / U) \otimes C_{P}(f) \otimes O(f)\right]>\lambda$. By Theorem 8 in [1], $\left[\Gamma_{P}(U, \tau / U) \otimes C_{P}(f)\right] \leq[\Gamma(f(U), \sigma / f(U))]$ and $\left[\tau\left(V^{\prime}\right) \otimes O(f)\right]=\max \left(0, \tau\left(V^{\prime}\right)+O(f)-1\right)=\max \left(0, \tau\left(V^{\prime}\right)+\bigwedge_{V \subseteq X} \min (1,1-\right.$ $\left.\left.\tau\left(V^{\prime}\right)+\sigma(f(V))\right)-1\right) \leq \max \left(0, \tau\left(V^{\prime}\right)+1-\tau\left(V^{\prime}\right)+\sigma(f(V))-1\right)=\sigma(f(V)) \leq$ $N_{f(x)}^{Y}\left(f\left(V^{\prime}\right)\right) \leq N_{f(x)}^{Y}(f(U))$.

Since $f$ is surjective, 


$$
\begin{aligned}
& L C(Y, \sigma)=L C(f(X), \sigma)=\bigwedge_{y \in f(x) \subseteq f(X)} \bigcup_{U^{\prime}=f(U) \subseteq f(X)}\left[N_{y}^{Y}\left(U^{\prime}\right) \otimes\left[\Gamma\left(U^{\prime}, \sigma / U^{\prime}\right)\right]\right. \\
& \geq \bigwedge_{y \in f(x) \subseteq f(X)}\left[N_{f(x)}^{Y}(f(U)) \otimes[\Gamma(f(U), \sigma / f(U))]\right. \\
& \geq \bigwedge_{y \in f(x) \subseteq f(X)}\left[\tau\left(V^{\prime}\right) \otimes O(f) \otimes \Gamma_{P}(U, \tau / U) \otimes C_{P}(f)\right] \geq \lambda .
\end{aligned}
$$

Theorem 4.7. Let $(X, \tau)$ and $(Y, \sigma)$ be two fuzzifying topological spaces and $f \in Y^{X}$ be surjective. Then $\models L_{P} C(X, \tau) \otimes I_{P}(f) \otimes O_{P}(f) \rightarrow$ $L_{P} C(Y, \sigma)$.

Proof. By Theorem 9 in [1], the proof is similar to the proof of Theorem 4.6 .

Theorems 4.6 and 4.7 are a generalization of the following corollary.

Corollary 4.2. Let $(X, \tau)$ and $(Y, \sigma)$ be two topological spaces and $f$ : $(X, \tau) \rightarrow(Y, \sigma)$ be surjective mapping. If $f$ is a pre-continuous (resp. preirresolute), open (resp. pre-open) and $X$ is locally strong compact, then $Y$ is locally compact (resp. locally strong compact) space.

Theorem 4.8. Let $\left\{\left(X_{s}, \tau_{s}\right): s \in S\right\}$ be a family of fuzzifying topological spaces, then

$=L_{P} C\left(\prod_{s \in S} X_{s}, \prod_{s \in S}\left(\tau_{P}\right)_{s}\right) \rightarrow \forall s\left(s \in S \wedge L_{P} C\left(X_{s},\left(\tau_{P}\right)_{s}\right) \wedge \exists T(T \sqsubset S \wedge \forall t(t \in\right.$ $\left.\left.S-T \wedge \Gamma_{P}\left(X_{t}, \tau_{t}\right)\right)\right)$.

Proof. It suffices to show that

$$
\begin{array}{r}
L_{P} C\left(\prod_{s \in S} X_{s}, \prod_{s \in S}\left(\tau_{P}\right)_{s}\right) \\
\leq \bigwedge_{s \in S}\left[L_{P} C\left(X_{s},\left(\tau_{P}\right)_{s}\right) \wedge \bigvee_{T \sqsubset S} \bigwedge_{t \in S-T} \Gamma_{P}\left(X_{t}, \tau_{t}\right)\right] .
\end{array}
$$

From Theorem 4.7 and Lemma 3.1 we have for any $t \in S$,

$$
\begin{gathered}
L_{P} C\left(\prod_{s \in S} X_{s}, \prod_{s \in S}\left(\tau_{P}\right)_{s}\right) \\
=\left[L_{P} C\left(\prod_{s \in S} X_{s}, \prod_{s \in S}\left(\tau_{P}\right)_{s}\right) \otimes C_{P}\left(p_{t}\right) \otimes O_{P}\left(p_{t}\right)\right] \leq L_{P} C\left(X_{t}, \tau_{t}\right) .
\end{gathered}
$$

So,

$$
\bigwedge_{t \in S-T} L_{P} C\left(X_{t}, \tau_{t}\right) \geq L_{P} C\left(\prod_{s \in S} X_{s}, \prod_{s \in S}\left(\tau_{P}\right)_{s}\right)
$$


By Theorem 3.2 we have

$$
\begin{aligned}
& \bigvee_{T \sqsubset S} \bigwedge_{t \in S-T} \Gamma_{P}\left(X_{t}, \tau_{t}\right) \\
\geq & {\left.\left[\bigvee_{U \subseteq \prod_{s \in S} X_{s}} \Gamma_{P}\left(U, \prod_{s \in S}\left(\tau_{P}\right)_{s} \backslash U\right) \otimes \bigvee_{X \subseteq \prod_{s \in S} X_{s}} N_{x}^{P^{X}}(U)\right)\right] } \\
\geq & \bigvee_{U \subseteq \prod_{s \in S} X_{s}} \bigvee_{\subseteq}\left[\prod_{s \in S} X_{s}\left(\Gamma_{P}\left(U, \prod_{s \in S}\left(\tau_{P}\right)_{s} \backslash U\right) \otimes N_{x}^{P^{X}}(U)\right)\right] \\
\geq & \left.\bigwedge_{X \subseteq \prod_{s \in S} X_{s}} \bigvee_{U \subseteq \prod_{s \in S} X_{s}}\left[\Gamma_{P}\left(U, \prod_{s \in S}\left(\tau_{P}\right)_{s} \backslash U\right) \otimes N_{x}^{P^{X}}(U)\right)\right] \\
= & L_{P} C\left(\prod_{s \in S} X_{s}, \prod_{s \in S}\left(\tau_{P}\right)_{s}\right) .
\end{aligned}
$$

Therefore,

$$
\begin{gathered}
L_{P} C\left(\prod_{s \in S} X_{s}, \prod_{s \in S}\left(\tau_{P}\right)_{s}\right) \\
\leq\left[\bigwedge_{t \in S-T} L_{P} C\left(X_{t}, \tau_{t}\right) \wedge \bigvee_{T \sqsubset S} \bigwedge_{t \in S-T} \Gamma_{P}\left(X_{t}, \tau_{t}\right)\right] .
\end{gathered}
$$

We can obtain the following corollary in crisp setting.

Corollary 4.3. Let $\left\{X_{\lambda}: \lambda \in \Lambda\right\}$ be a family of nonempty topological spaces. If $\prod_{\lambda \in \Lambda} X_{\lambda}$ is locally strong compact, then each $X_{\lambda}$ is locally strong compact and all but finitely many $X_{\lambda}$ are strong compact

Conclusion: The present paper investigates topological notions when these are planted into the framework of Ying's fuzzifying topological spaces (in semantic method of continuous valued-logic). It continue various investigations into fuzzy topology in a legitimate way and extend some fundamental results in general topology to fuzzifying topology. An important virtue of our approach (in which we follow Ying) is that we define topological notions as fuzzy predicates (by formulae of Łukasiewicz fuzzy logic) and prove the validity of fuzzy implications (or equivalences). Unlike the (more 
wide-spread) style of defining notions in fuzzy mathematics as crisp predicates of fuzzy sets, fuzzy predicates of fuzzy sets provide a more genuine fuzzification; furthermore the theorems in the form of valid fuzzy implications are more general than the corresponding theorems on crisp predicates of fuzzy sets. The main contributions of the present paper are to give characterizations of fuzzifying strong compactness. Also, we define the concept of locally strong compactness of fuzzifying topological spaces and obtain some basic properties of such spaces. There are some problems for further study:

(1) One obvious problem is: our results are derived in the Eukasiewicz continuous logic. It is possible to generalize them to more general logic setting, like residuated lattice-valued logic considered in [34-35].

(2) What is the justification for fuzzifying locally strong compactness in the setting of $(2, \mathrm{~L})$ topologies.

(3) What is the justification for fuzzifying locally strong compactness in $(M, L)$-topologies etc.

\section{Acknowledgments}

This paper was presented in the 6th International Conference for Young Scientists in Basic and Applied Sciences ICYS-BAS19, 27-30 March 2019, Hawaii Le Jardin Resort, Hurghada-Egypt.

\section{References}

[1] S. A. Abd El-Baki and O. R. Sayed, Pre-irresolutness and strong compactness in fuzzifying topology, Journal of the egyptian mathematical society, vol. 15 (1), pp. 41-56, 2007. [On line]. Available: https:/ / bit.ly/ 3r1j9UR

[2] K. M. Abd El-Hakeim, F. M. Zeyada, and O. R. Sayed, "Pre-Continuity and $\mathrm{D}(\mathrm{c}, \mathrm{p})$-continuity in fuzzifying topology", Fuzzy sets and systems, vol. 119, no. 3, pp. 459-471, 2001. doi: 10.1016/ s0165-0114(99)00097-4

[3] K. M. Abd El-Hakeim, F. M. Zeyada and O. R. Sayed, "Pre-separation axioms in fuzzifying topology, Fuzzy systems and mathematics, vol. 17, pp. 29-37, 2003.

[4] C. L. Chang, "F uzzy topological spaces", J ournal of mathematical analysis and applications, vol. 24, pp. 182-190, 1968. [On line]. A vailable: https:// bit.ly/ 31G CIOX

[5] A. Dutta and B. C. Tripathy, "On fuzzy b- $\theta$ open sets in fuzzy topological space”, Journal of intelligent \& fuzzy systems, vol. 32, no. 1, pp. 137-139, 2017. doi: $10.3233 /$ jifs-151233 
[6] J. A. Goguen, "The fuzzy tychonoff theorem", Journal of mathematical analysis and applications, vol. 43, no. 3, pp. 734-742, 1973. doi: 10.1016/ 0022-247x(73)90288-6

[7] U. Höhle, "Upper semicontinuous fuzzy sets and applications”, Journal of mathematical analysis and applications, vol. 78, no. 2, pp. 659-673, 1980. doi: 10.1016/ 0022-247x(80)90173-0

[8] U. Höhle, Many valued topology and its applications. Dordrecht: Kluwer Academic Publishers, 2001.

[9] U. Höhle and S. E. Rodabaugh, Mathematics of fuzzy sets: logic, topology, and measure theory. Dordrecht: Kluwer Academic Publishers, 1999. doi:10.1007/ 978-1-4615-5079-2

[10] U. Höhle, S. E. Rodabaugh, and A. Sostak, (Eds.), Fuzzy Topology. Elsevier, 1995. https:/ / bit.ly/ 3rJ3sSB

[11] Höhle U and A. Šostak, "Axiomatic foundations of fixed-basis fuzzy topology," in Mathematics of fuzzy sets: logic, topology, and measure theory, Höhle U and S. E. Rodabaugh, Eds. Dordrecht: Kluwer Academic Publishers, 1999, pp. 123-272. doi: 10.1007/978-1-4615-5079-2_5

[12] J. L. Kelley, General Topology. New York: Van Nostrand, 1955.

[13] Kubiak, T., 1985. On fuzzy topologies. Ph.D. Thesis. Adam Mickiewicz University, Poznan, Poland.

[14] Y. M. Liu and M. K. Luo, Fuzzy topology. Singapore: World Scientific, 1998.

[15] A. S. Mashhour, M. E. Abd El-Monsef and S. N. El-Deeb, "On precontinuous and weak pre-continuous mappings", Proceedings of the mathematical and physical society of Egypt, vol. 53, pp. 255-263, 1982.

[16] A. S. Mashhour, M. E. Abd El-Monsef, I. A. Hasanein and T. Noiri, "Strongly compact spaces", Delta journal of science, vol. 8, pp. 30-64, 1984.

[17] D. Qiu, "Fuzzifying topological linear spaces", Fuzzy sets and systems, vol. 147, no. 2, pp. 249-272, 2004. doi: 10.1016/j.fss.2003.10.024

[18] D. Qiu, "Characterizations of fuzzy finite automata", Fuzzy sets and systems, vol. 141, no. 3, pp. 391-414, 2004. doi: 10.1016/s01650114(03)00202-1 
[19] G. C. Ray and B. C. Tripathy, "Fuzzy $\delta *$-almost continuous and fuzzy $\delta *$-continuous functions in mixed fuzzy ideal topological spaces", Proyecciones (Antofagasta), vol. 39, no. 2, pp. 435-449, 2020. doi: 10.22199/ issn.0717-6279-2020-02-0027

[20] S. E. Rodabaugh, "Categorical foundations of variable-basis fuzzy topology", in Mathematics of fuzzy sets: logic, topology, and measure theory, Höhle U and S. E. Rodabaugh, Eds. Dordrecht: Kluwer Academic Publishers, 1999, pp. 273-388. doi: 10.1007/978-1-4615-5079-2_6

[21] J. B. Rosser and A. R. Turquette, Many-valued logics. Amsterdam: North-Holland, 1952.

[22] D. J. Sarma and B. C. Tripathy, "Fuzzy semi-pre quasi neighborhood structure”, Afrika matematika, vol. 30, no. 1-2, pp. 217-221, 2019. doi: 10.1007/ s13370-018-0637-6

[23] O. R. Sayed, On fuzzifying topological spaces. Ph.D. Thesis. Assiut University, Egypt, 2002

[24] J. Shen, "Locally compactness in fuzzifying topology", Journal of fuzzy mathematics, vol. 2, no. 4, pp. 695-711, 1994.

[25] B. C. Tripathy and G. C. Ray, "Mixed fuzzy ideal topological spaces", Applied mathematics and computation, vol. 220, pp. 602-607, 2013. doi: 10.1016/j.amc.2013.05.072

[26] B. C. Tripathy and G. C. Ray, "On $\delta$-continuity in mixed fuzzy topological spaces”, Boletim da sociedade paranaense de matemática, vol. 32, no. 2, pp. 175-187, 2014. doi: 10.5269/ bspm.v32i2.20254

[27] B. C. Tripathy and G. C. Ray, "Weakly continuous functions on mixed fuzzy topological spaces", Acta scientiarum. technology, vol. 36, no. 2, pp. 331-335, 2014. doi: 10.4025/ actascitechnol.v36i2.16241

[28] B. C. Tripathy and G. C. Ray, "Fuzzy $\delta$-I -continuity in mixed fuzzy ideal topological spaces", Journal of applied analysis, vol. 24, no. 2, pp. 233-239, 2018. doi: 10.1515/ jaa-2018-0022

[29] G. J. Wang, Theory of L-fuzzy topological spaces. Xi'an: Shanxi Normal University Press, 1988. (in Chinese).

[30] M. S. Ying, "A new approach for fuzzy topology (I)", Fuzzy sets and systems, vol. 39, no. 3, pp. 303-321, 1991. doi: 10.1016/0165-0114 (91)90100-5 
[31] M. S. Ying, "A new approach for fuzzy topology (II)", Fuzzy sets and systems, vol. 47, no. 2, pp. 221-232, 1992. doi: 10.1016/ 0165-0114(92) 90181-3

[32] M. S. Ying, "A new approach for fuzzy topology (III)", Fuzzy sets and systems, vol. 55, no. 2, pp. 193-207, 1993. doi: 10.1016/ 0165-0114(93) 90132-2

[33] M. S. Ying, "Compactness in fuzzifying topology", Fuzzy sets and systems, vol. 55, no. 1, pp. 79-92, 1993. doi: 10.1016/ 0165-0114(93) 90303-y

[34] M. S. Ying, "Fuzzifying topology based on complete residuated lattice-valued logic (I)", Fuzzy sets and systems, vol. 56, no. 3, pp. 337-373, 1993. doi: 10.1016/ 0165-0114(93)90215-4

[35] M. S. Ying, "Fuzzy topology based on residuated lattice-valued logic," Acta mathematica sinica, English series, vol. 17, no. 1, pp. 89-102, 2001. doi: $10.1007 /$ s101140000079

\section{O. R. Sayed}

Department of Mathematics, Faculty of Science,

Assiut University, Assiut 71516,

Egypt

e-mail: o_r_sayed@yahoo.com

Corresponding author

and

\section{Adem Kiliçman}

Department of Mathematics, University Putra Malaysia, 43400 UPM, Serdang, Selangor,

Malaysia

e-mail: akilic@upm.edu.my 